TRANSACTIONS OF THE

AMERICAN MATHEMATICAL SOCIETY

Volume 350, Number 10, October 1998, Pages 4167-4193

S $0002-9947(98) 02125-4$

\title{
THE STRUCTURE OF INDECOMPOSABLE INJECTIVES IN GENERIC REPRESENTATION THEORY
}

\author{
GEOFFREY M. L. POWELL
}

\begin{abstract}
This paper considers the structure of the injective objects $I_{V_{n}}$ in the category $\mathcal{F}$ of functors between $\mathbb{F}_{2}$-vector spaces. A co-Weyl object $J_{\lambda}$ is defined, for each simple functor $F_{\lambda}$ in $\mathcal{F}$. A functor is defined to be $J$-good if it admits a finite filtration of which the quotients are co-Weyl objects. Properties of $J$-good functors are considered and it is shown that the indecomposable injectives in $\mathcal{F}$ are $J$-good. A finiteness result for proper sub-functors of coWeyl objects is proven, using the polynomial filtration of the shift functor $\tilde{\Delta}: \mathcal{F} \rightarrow \mathcal{F}$. This research is motivated by the Artinian conjecture due to Kuhn, Lannes and Schwartz.
\end{abstract}

\section{INTRODUCTION}

The purpose of this paper is to study the structure of the indecomposable injective objects in the category $\mathcal{F}$ of functors $\mathcal{E}_{f} \rightarrow \mathcal{E}$, where $\mathcal{E}$ is the category of $\mathbb{F}$-vector spaces and $\mathcal{E}_{f}$ the full sub-category of finite dimensional spaces. The field $\mathbb{F}$ is taken to be a finite field and, for the purposes of this introduction and for some of the main results, this is taken to be $\mathbb{F}_{2}$, the prime field with two elements. Kuhn has termed $\mathcal{F}$ the category of generic representations in his sequence of papers [K1, K2, K3]. The special interest of this category of functors in the finite field case follows from the link with the category of unstable modules over the Steenrod algebra. A review of the category $\mathcal{F}$ is presented in Section 2, to which the reader is referred for any unexplained terminology.

The paper shows that a high degree of understanding of the structure of the indecomposable injective functors in $\mathcal{F}$ is obtained by concentrating on certain smaller building blocks, which the author has named co-Weyl objects, by analogy with the theory of highest weight categories, introduced by Cline, Parshall and Scott in [CPS]. It is readily seen that $\mathcal{F}$ does not have the structure of a highest weight category; however, many of the features of such a category may be recovered by new techniques.

The simple functors in $\mathcal{F}$ correspond to simple $G L_{n}(\mathbb{F})$ modules, as $n$ varies. A co-Weyl object is associated to each simple functor: if $F_{\lambda}$ is a simple functor indexed by $\lambda$, then the corresponding co-Weyl object is written as $J_{\lambda}$. This is the largest analytic functor such that $J_{\lambda}$ has simple socle $F_{\lambda}$ and $J_{\lambda}\left(\mathbb{F}^{n}\right)=F_{\lambda}\left(\mathbb{F}^{n}\right)$, where $n$ is the smallest integer such that $F_{\lambda}\left(\mathbb{F}^{n}\right) \neq 0$.

Received by the editors December 5, 1996.

1991 Mathematics Subject Classification. Primary 18G05, 20G40, 55S10.

The author was supported by a Royal Society (GB) ESEP fellowship at the Institut Galilée, Université de Paris-Nord, France during the preparation of this work. The final version was prepared whilst the author was a visitor at the University of Virginia, Charlottesville. 
A functor $F \in \mathcal{F}$ is said to be $J$-good if it has a finite filtration of which the quotients are co-Weyl objects. The justification for interest in such functors is provided by the first important result of this paper, which is proved in Section 4; it may be regarded as saying that the co-Weyl objects are the building blocks for the category $\mathcal{F}$.

Theorem 1. The indecomposable injective functors in $\mathcal{F}$ are $J$-good.

The proof of this result deserves some comment; recall (see Section 2) that $I_{V}$ denotes a standard injective in $\mathcal{F}$, that $\mathcal{M}_{n}$ is the category of $\operatorname{End}\left(\mathbb{F}^{n}\right)$-modules and that $\mathcal{G} \mathcal{L}_{n}$ denotes the category of $\operatorname{Aut}\left(\mathbb{F}^{n}\right)$-modules, which may be considered as a full sub-category of $\mathcal{M}_{n}$. The evaluation functor $E_{n}: \mathcal{F} \rightarrow \mathcal{M}_{n},\left(F \mapsto F\left(\mathbb{F}^{n}\right)\right)$ admits a right adjoint $R_{n}: \mathcal{M}_{n} \rightarrow \mathcal{F}$. The functor $R_{n}$ is not exact; however, it is shown in Section 2 that the restriction of $R_{n}$ to $\mathcal{G} \mathcal{L}_{n}$ is exact. This is the key step in the proof that the functors $I_{V}$ are $J$-good, since it may be shown that $J_{\lambda} \cong R_{n}\left(S_{\lambda}\right)$, where $n$ is the least integer such that $F_{\lambda}\left(\mathbb{F}^{n}\right)$ is non-zero and $S_{\lambda}$ is the simple $G L_{n}$-module $F_{\lambda}\left(\mathbb{F}^{n}\right)$.

The proof of the theorem then requires the use of certain properties of the category of $J$-good functors, in particular that a direct summand of a $J$-good functor is again $J$-good. The proof of this result requires an estimate of the size of co-Weyl functors. This is achieved by using the next key ingredient of the paper, derived from the study of the polynomial filtration of the shift functor $\tilde{\Delta}: \mathcal{F} \rightarrow \mathcal{F}$, which was introduced in $[\mathrm{P}]$. This gives a collection of functors $\tilde{\nabla}_{n}$, which are linked by natural surjections $\tilde{\nabla}_{n} \rightarrow \tilde{\nabla}_{n+1}$. These functors have some good properties; in particular, they preserve injections and surjections, although they are not exact. This paper shows that the concept of $\tilde{\nabla}_{n}$-nilpotence is important: a functor $F$ is said to be $\tilde{\nabla}_{n}$-nilpotent if there exists an integer $k$ such that $\left(\tilde{\nabla}_{n}\right)^{k} F=0$. This provides the required estimate of the size of a co-Weyl object: if $J_{\lambda}=R_{n}\left(S_{\lambda}\right)$, where $S_{\lambda}$ is a simple $\mathcal{G} \mathcal{L}_{n}$-module, then $J_{\lambda}$ is not $\tilde{\nabla}_{n}$-nilpotent, whereas $\tilde{\nabla}_{n+1} J_{\lambda}=0$.

The theorem may be used to show that the Cartan invariants $\operatorname{dim} \operatorname{Hom}_{\mathcal{F}}\left(I_{\lambda}, I_{\mu}\right)$ in the category $\mathcal{F}$ determine the multiplicity of co-Weyl functors in $J$-good filtrations of the indecomposable injectives. In particular, this gives a proof of the following result:

Corollary 2. The Cartan matrix for $\mathbb{F}\left[M_{n}\right]$ determines the Cartan matrix for $\mathbb{F}\left[G L_{n}\right]$.

In addition, the author has determined an homological characterization of $J$ good functors, based on the fact that the indecomposable injectives in $\mathcal{F}$ are $J$ good. This was inspired by results in algebraic group theory related to the notion of good filtration dimensions (see for example $[\mathrm{FP}]$ or $[\mathrm{J}]$ ). In the statement of the result below (and throughout the paper) $V_{d}$ represents the $\mathbb{F}$-vector space of dimension $d$ and $I_{V_{d}}$ is the associated injective.

Theorem 3. An analytic functor $F$ with finite socle is $J$-good if and only if there is an integer $d$ such that $F$ has an injective resolution $0 \rightarrow F \rightarrow I^{\bullet}$, in which each $I^{k}$ is a finite direct sum $\bigoplus I_{V_{d}}$.

This result is proved in Section 5 and plays a pivotal role in the paper; it implies immediately that the tensor product of $J$-good functors is $J$-good and also that the difference functor of a $J$-good functor is $J$-good. This shows that $\tilde{\nabla}_{n} J_{\lambda}$ occurs as 
a quotient of a $J$-good functor, namely $\Delta J_{\lambda}$. This is a major input into Proposition 6.0.4, which shows that $\tilde{\nabla}_{n} J_{\lambda} \cong J_{\lambda}$ (except in one special case), when $n$ is the least integer such that $J_{\lambda}\left(\mathbb{F}^{n}\right) \neq 0$.

This makes the following result striking; it appears as Theorem 6.0.1. The author regards this as the most important result of the paper, since it yields considerable information about the structure of the injectives in $\mathcal{F}$.

Theorem 4. Suppose that $J_{\lambda}$ is a co-Weyl functor induced from a simple $G L_{n^{-}}$ module. If $F \hookrightarrow J_{\lambda}$ is a proper sub-functor, then $F$ is $\tilde{\nabla}_{n}$-nilpotent.

The author has termed this result the Simplicity Theorem, since it implies that $J_{\lambda}$ is a simple object in the abelian category $\mathcal{F}_{\omega} / \tilde{\nabla}_{n}-\mathcal{N} i l$ of analytic functors localized away from the full sub-category of $\tilde{\nabla}_{n}$-nilpotent functors. In a future paper, the author will exploit this theorem to show how this implies certain results about extension groups in $\mathcal{F}$, which have interesting corollaries for the category of unstable modules over the Steenrod algebra.

Section 7 briefly discusses the category $\mathcal{B}^{\mathcal{E}_{f}}$ of Boolean algebra valued functors, motivated by the fact that these correspond (under the work of [HLS]) to unstable algebras over the Steenrod algebra. It is shown that:

Theorem 5. If $K \in \mathcal{B}^{\mathcal{E}_{f}}$ has finite socle, then $K$ is $J$-good as a functor in $\mathcal{F}$.

This result shows that many of the functors in $\mathcal{F}$ which arise from the cohomology of a space are $J$-good; the hypothesis essentially restricts us to the study of algebras of finite transcendence degree, which take finite-dimensional values.

1.1. Remarks on the odd prime case. Most of the results discussed above may be extended to the odd primary case, namely the case where $\mathbb{F}=\mathbb{F}_{p}$, where $p$ is an odd prime. The essential change is that now one uses the fact that $\tilde{\nabla}_{(p-1) n} I_{V_{n}} \cong I_{V_{n}}$ and $\tilde{\nabla}_{(p-1) n+1} I_{V_{n}}=0$.

1.2. Concerning the Artinian conjecture. In this section, the implication of the results of this paper for the study of the conjecture of the title is explained; again it is supposed that $\mathbb{F}$ is the field $\mathbb{F}_{2}$. Recall that $\mathcal{F}$ is an abelian category; an object $F \in \mathcal{F}$ is said to be Artinian if every descending sequence of sub-functors of $F$ stabilizes. The following has been conjectured; it appears in print in [K1] and is attributed largely to Lionel Schwartz, in conjunction with Nick Kuhn and Jean Lannes.

The Artinian conjecture: The functors $I_{V_{n}}$ are Artinian.

An affirmative resolution of this conjecture would have the consequence that an analytic functor $F$ with finite socle would have an injective resolution $0 \rightarrow F \rightarrow I^{\bullet}$, in which each $I^{k}$ has finite socle. The calculation of the extension groups $\operatorname{Ext}_{\mathcal{F}}(-,-)$ is related to the study of Mac Lane cohomology with coefficients in twisted bifunctors of coefficients (see [FLS]) and with topological Hochschild cohomology; therefore the knowledge of such a finiteness result for injective resolutions would imply finiteness results for these theories, at least when the underlying ring is a finite field.

A stronger formulation of the conjecture may be given, using the following terminology. A functor $F$ is said to be simple Artinian of type 0 if it is a simple functor; $F$ is Artinian of type $n$ if it has a finite filtration of which the sub-quotients are 
simple Artinian of type $n$. Finally, $F$ is simple Artinian of type $n$ if every proper sub-functor of $F$ is Artinian of type $n-1$.

The strong Artinian conjecture: The functors $I_{V_{n}}$ are Artinian of type $n$.

This conjecture is known to be true for the functors $I_{\mathbb{F}}$, for any finite field $\mathbb{F}$. For $\mathbb{F}=\mathbb{F}_{2}$, the only other case known is $I_{\mathbb{F}^{2}}$, which was shown by the author to be Artinian of type two, in [PS]. The results of this paper, together with the methods of [PS] lead us to propose:

The very strong Artinian conjecture: If $S_{\lambda}$ is a simple $G L_{n}$-module, then the associated co-Weyl object $J_{\lambda}=R_{n} S_{\lambda}$ is simple Artinian of type $n$.

It is not difficult to deduce that the very strong conjecture would imply the strong conjecture. The simplicity theorem of this paper implies the following reduction:

Proposition 1.2.1. The very strong Artinian conjecture holds for all $J_{\lambda}$ induced from simple $G L_{n}$-modules if and only if the strong Artinian conjecture holds for $I_{V_{n-1}}$ and every sub-functor of $I_{V_{n}}$ which is $\tilde{\nabla}_{n}$-nilpotent is Artinian of type $n-1$.

This is of interest, since the largest sub-functor $F$ of $I_{V_{n}}$ with $\left(\tilde{\nabla}_{n}\right)^{t} F=0$ is the kernel of an explicit map

$$
I_{V_{n}} \rightarrow I_{V_{n+t}}
$$

which is induced by an element $\mathbb{F}\left[\operatorname{hom}\left(V_{n}, V_{n+t}\right)\right]$ which is described in $[\mathrm{P}]$. It follows that the very strong Artinian conjecture may be reduced to studying certain explicit 'small' sub-functors of $I_{V_{n}}$

\section{The CATEgory $\mathcal{F}$}

2.1. Review of the category $\mathcal{F}$. The category $\mathcal{F}$ is abelian, with structure induced from the abelian category $\mathcal{E}$ of $\mathbb{F}$-vector spaces. In particular, a sequence $F \rightarrow G \rightarrow H$ of functors is short exact if and only if it is short exact when evaluated on any finite dimensional vector space. The usual definitions of simple object and composition series are taken. The classification of the simple objects is reviewed in Section 2.3. Good references for this material are the papers [K1, K2, K3], together with the first half of the book by Lionel Schwartz [S].

The category $\mathcal{F}$ is equipped with a duality functor, $D: \mathcal{F} \rightarrow \mathcal{F}^{o p}$, which generalizes the notion of transpose duality in representation theory. This is defined by $D F(V):=F\left(V^{*}\right)^{*}$, where the $*$ denotes the vector space dual. If $F$ takes finite dimensional values, then it follows that there is a natural isomorphism $D D F \cong F$.

A functor $F \in \mathcal{F}$ is said to be finite if it has a finite composition series and is said to be analytic if it is the colimit of its finite sub-objects. The full sub-category of $\mathcal{F}$ with objects which are the analytic functors is denoted by $\mathcal{F}_{\omega}$ and is termed the category of analytic functors.

The category $\mathcal{F}$ has enough injectives and projectives; the standard projective $P_{V}$ is defined by $P_{V}\left(\mathbb{F}^{n}\right)=\mathbb{F}\left[\operatorname{hom}\left(V, \mathbb{F}^{n}\right)\right]$, where $V$ is a finite dimensional vector space. Up to isomorphism, this is determined by the representing property:

$$
\operatorname{Hom}_{\mathcal{F}}\left(P_{V}, F\right) \cong F(V) \text {, }
$$

naturally in the functor $F$. The standard injective $I_{V}$ may be defined as the dual $D P_{V}$ of the projective. Explicitly, this means that:

$$
I_{V}\left(\mathbb{F}^{n}\right)=\mathbb{F}^{\operatorname{hom}\left(V, \mathbb{F}^{n *}\right)} .
$$


Again, up to isomorphism, this is determined by a co-representing property, which is dual to that given above. From this discussion it is clear that:

$$
\operatorname{Hom}_{\mathcal{F}}\left(I_{V}, I_{W}\right) \cong \mathbb{F}[\operatorname{hom}(V, W)],
$$

so that one may regard $I_{-}$as a covariant functor from $\mathcal{E}_{f}$ to $\mathcal{F}$.

The injective functors $I_{V}$ are analytic, whereas $P_{V}$ is not analytic in general. In fact, $P_{V}$ splits as $\mathbb{F} \oplus \widehat{P_{V}}$ and $\widehat{P_{V}}$ has no finite sub-objects.

The category $\mathcal{F}$ is equipped with the shift functor $\tilde{\Delta}: \mathcal{F} \rightarrow \mathcal{F}$, which is defined on objects by $\tilde{\Delta} F(V)=F(V \oplus \mathbb{F})$. This functor is right adjoint to the functor $-\otimes P_{\mathbb{F}}$. The functor $\tilde{\Delta}$ splits naturally as $\tilde{\Delta} \cong \Delta \oplus \mathrm{id}$, where the splitting is given by the natural split injection $F(V) \hookrightarrow F(V \oplus \mathbb{F})$; the functor $\Delta$ is known as the difference functor.

An important concept in $\mathcal{F}$ is that of the polynomial degree of a functor, which has been studied since the work of Eilenberg and Mac Lane [EM]. The definition of a polynomial functor taken here uses the difference functor. Namely, a functor $F$ is said to be polynomial of degree $\leq d$ if $\Delta^{d+1} F=0$. The full sub-category of polynomial functors of degree $\leq d$ is denoted by $\mathcal{F}_{d}$. The inclusion $\mathcal{F}_{d} \hookrightarrow \mathcal{F}$ admits a left adjoint $q_{d}: \mathcal{F} \rightarrow \mathcal{F}_{d}$ and a right adjoint $p_{d}: \mathcal{F} \rightarrow \mathcal{F}_{d}$. These are related by a natural isomorphism $D q_{d} F \cong p_{d} D F$.

The shift functor $\tilde{\Delta}$ admits a polynomial filtration, which was introduced in $[\mathrm{P}]$. This may be illustrated by observing that there is an isomorphism:

$$
\tilde{\Delta} I_{V} \cong \mathbb{F}^{V^{*}} \otimes I_{V} .
$$

Here $\mathbb{F}^{V^{*}}$ may be regarded as the free $\mathbb{F}$-Boolean algebra on a vector space basis of $V$ and is a constant functor. This admits the usual notion of a polynomial filtration, which induces a filtration of $\tilde{\Delta} I_{V}$.

Formally, one may define the functor $\left[p_{k} \tilde{\Delta}\right]$ to be the right adjoint to the functor $-\otimes q_{k} P_{\mathbb{F}}$; by convention take $\left[p_{-1} \tilde{\Delta}\right]=0$. It is useful to study the quotients $\tilde{\nabla}_{s}$ for $s \geq 0$ and sub-quotients $\nabla_{s}$ defined below:

Definition 2.1.1. Suppose $s \geq 0$.

1. $\tilde{\nabla}_{s}:=\tilde{\Delta} /\left[p_{s-1} \tilde{\Delta}\right]$.

2. $\nabla_{s}:=\left[p_{s} \tilde{\Delta}\right] /\left[p_{s-1} \tilde{\Delta}\right]$.

The functors $\tilde{\nabla}_{s}: \mathcal{F} \rightarrow \mathcal{F}$ are the natural functors to be considering, although it can be useful in applications to use the functors $\nabla_{s}$. One should observe that $\tilde{\nabla}_{1}$ identifies with the difference functor $\Delta$; moreover, there are natural surjections $\tilde{\nabla}_{s} \rightarrow \tilde{\nabla}_{s+1}$, for each $s$. The essential properties of these functors are summarized below:

Theorem 2.1.2. $[\mathrm{P}]$

1. The functors $\tilde{\nabla}_{s}$ preserve injections, surjections and direct sums.

2. If $F$ is an analytic functor with finite socle then $\tilde{\nabla}_{s} F$ is an analytic functor with finite socle.

3. The functor $\tilde{\nabla}_{s} I_{V}$ is injective.

4. If $\mathbb{F}=\mathbb{F}_{2}$, then $\nabla_{s} I_{V} \cong \Lambda^{s}(V) \otimes I_{V}$; if $g: V \rightarrow W$ is a map of vector spaces which induces $[g]: I_{V} \rightarrow I_{W}$, then $\nabla_{s} g$ identifies with $\Lambda^{s}(g) \otimes[g]$ : $\Lambda^{s}(g) \otimes I_{V} \rightarrow \Lambda^{s}(W) \otimes I_{W}$. 
2.2. Recollements in $\mathcal{F}$. Throughout this paper, $\mathcal{M}_{n}$ denotes the category of left $M_{n}=\operatorname{End}\left(\mathbb{F}^{n}\right)$-modules and $\mathcal{G} \mathcal{L}_{n}$ is the category of left $G L_{n}$-modules, where $G L_{n}$ is the group $\operatorname{Aut}\left(\mathbb{F}^{n}\right)$. The canonical inclusion $\operatorname{Aut}\left(\mathbb{F}^{n}\right) \subset \operatorname{End}\left(\mathbb{F}^{n}\right)$ induces an inclusion $\mathbb{F}\left[G L_{n}\right] \hookrightarrow \mathbb{F}\left[M_{n}\right]$, which is split by the ring map $\mathbb{F}\left[M_{n}\right] \rightarrow \mathbb{F}\left[G L_{n}\right]$ which sends singular endomorphisms to zero and automorphisms to themselves.

Kuhn has stressed in [K2] that a fundamental tool for studying the link between the representation theory of the above rings is the recollement diagram:

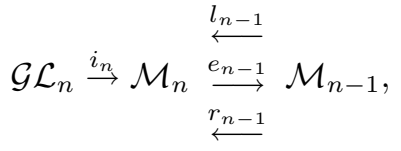

in which $e_{n-1}: \mathcal{M}_{n} \rightarrow \mathcal{M}_{n-1}$ is induced by a projection $e_{n-1} \in \operatorname{End}\left(V_{n}\right)$ onto $\mathbb{F}^{n-1}$, via $M \mapsto e_{n-1} M$. The functor $l_{n-1}$ is the left adjoint to $e_{n-1}$ and $r_{n-1}$ is the right adjoint to $e_{n-1}$. These functors are given on objects $N \in \mathcal{M}_{n-1}$ by

$$
\begin{aligned}
l_{n-1} N & :=\mathbb{F}\left[\operatorname{hom}\left(\mathbb{F}^{n-1}, \mathbb{F}^{n}\right)\right] \otimes_{M_{n-1}} N, \\
r_{n-1} N & :=\operatorname{Hom}_{M_{n-1}}\left(\mathbb{F}\left[\operatorname{hom}\left(\mathbb{F}^{n}, \mathbb{F}^{n-1}\right)\right], N\right) .
\end{aligned}
$$

There are related recollement diagrams for the category $\mathcal{F}$; write $E_{n}: \mathcal{F} \rightarrow \mathcal{M}_{n}$ for the evaluation functor $F \mapsto F\left(\mathbb{F}^{n}\right)$ and $\mathcal{F}^{n+1}$ for the kernel of $E_{n}$, which is the full sub-category of functors $F$ with $F\left(\mathbb{F}^{n}\right)=0$. There is a recollement diagram:

$$
\mathcal{F}^{n+1} \rightarrow \mathcal{F} \underset{\stackrel{E_{n}}{\stackrel{E_{n}}{\leftrightarrows}}}{\stackrel{L_{n}}{\leftrightarrows}} \mathcal{M}_{n},
$$

where $L_{n}$ and $R_{n}$ are given by

$$
\begin{aligned}
L_{n} M & :=P_{V_{n}} \otimes_{M_{n}} M, \\
R_{n} M & :=\operatorname{Hom}_{M_{n}}\left(\mathbb{F}\left[\operatorname{hom}\left(-, V_{n}\right)\right], M\right) .
\end{aligned}
$$

These functors are related via the duality functor, restricted to a functor $D: \mathcal{M}_{n} \rightarrow$ $\mathcal{M}_{n}^{o p}$, which identifies with transpose duality:

$$
D L_{n} M \cong R_{n} D M .
$$

The right adjoint to the inclusion $\mathcal{F}^{n+1}=\operatorname{Ker} E_{n} \hookrightarrow \mathcal{F}$ is also used throughout the paper; this is denoted by $k_{n}: \mathcal{F} \rightarrow \mathcal{F}^{n+1}$ and is defined on objects by $k_{n}(F):=$ ker $\left\{F \mapsto R_{n} E_{n} F\right\}$, where the map is the unit of the $\left(E_{n}, R_{n}\right)$-adjunction.

The functor $E_{n}: \mathcal{F} \rightarrow \mathcal{M}_{n}$ restricts to the category $\mathcal{F}^{n}$ to give a functor which factorizes as $\mathcal{F}^{n} \rightarrow \mathcal{G} \mathcal{L}_{n} \stackrel{i_{n}}{\rightarrow} \mathcal{M}_{n}$, so that $\left.E_{n}\right|_{\mathcal{F}^{n}}$ may be regarded as a functor $E_{n}: \mathcal{F}^{n} \rightarrow \mathcal{G} \mathcal{L}_{n}$, which fits into the restricted recollement diagram:

$$
\mathcal{F}^{n+1} \rightarrow \mathcal{F}^{n} \underset{\stackrel{L_{n}}{\stackrel{E_{n}}{\rightleftarrows}} \mathcal{G}}{\stackrel{L_{n}}{\longleftarrow}} \mathcal{G} \mathcal{L}_{n} .
$$

The important fact about the above functors is given by

Proposition 2.2.1. The functors $R_{n}, L_{n}: \mathcal{G} \mathcal{L}_{n} \rightarrow \mathcal{F}^{n}$ are exact. 
Proof. Using the duality relation between $L_{n}$ and $R_{n}$, it suffices to prove that the functor $L_{n}: \mathcal{G} \mathcal{L}_{n} \rightarrow \mathcal{F}^{n}$ is exact. Recall that a $G L_{n}$-module is regarded as an object in $\mathcal{M}_{n}$ by using the functor $i_{n}: \mathcal{G} \mathcal{L}_{n} \rightarrow \mathcal{M}_{n}$, so that $M$ is regarded as an $\mathbb{F}\left[\right.$ End $\left.V_{n}\right]$ module via the canonical map $\mathbb{F}\left[\right.$ End $\left.V_{n}\right] \rightarrow \mathbb{F}\left[G L_{n}\right]$. Also, by definition, $L_{n} M$ is isomorphic to the functor $\mathbb{F}\left[\operatorname{hom}\left(V_{n},-\right)\right] \otimes_{\operatorname{End} V_{n}} M$.

Since singular endomorphisms act trivially upon $M$, this identifies with the functor $\mathbb{F}\left[\operatorname{inj}\left(V_{n},-\right)\right] \otimes_{G L_{n}} M$, where $\operatorname{inj}\left(V_{n}, W\right)$ denotes the set of injections from $V_{n}$ to $W$ and $\mathbb{F}\left[\operatorname{inj}\left(V_{n},-\right)\right]$ is given the structure of a functor by regarding it as a quotient of $\mathbb{F}\left[\operatorname{hom}\left(V_{n},-\right)\right]$. Now, as a right $G L_{n}$-set, $\operatorname{inj}\left(V_{n}, W\right)$ identifies as $\operatorname{Gr}_{n}(W) \times G L_{n}$, the free $G L_{n}$-set, where $\operatorname{Gr}_{n}(W)$ is the Grassmannian of $n$ dimensional planes in $W$. Hence, $\mathbb{F}\left[\operatorname{inj}\left(V_{n}, W\right)\right]$ is free as a right $G L_{n}$-module, namely $\mathbb{F}\left[\operatorname{inj}\left(V_{n}, W\right)\right] \cong \mathbb{F}\left[\operatorname{Gr}_{n}(W)\right] \otimes \mathbb{F}\left[G L_{n}\right]$. (Note that this is not intended to be an isomorphism of $\operatorname{End}(W)$-modules.)

In particular, this gives that

$$
L_{n} M(W) \cong \mathbb{F}\left[\operatorname{inj}\left(V_{n}, W\right)\right] \otimes_{G L_{n} M} \cong \mathbb{F}\left[G r_{n}(W)\right] \otimes M,
$$

as vector spaces. The functor $\mathbb{F}\left[G r_{n}(W)\right] \otimes-$ is exact for each $W$, as a functor $\mathcal{G} \mathcal{L}_{n} \rightarrow \mathcal{E}_{f}$, so that the functor $L_{n}$ is exact, as required, since exactness is only checked at the level of vector spaces.

Remark 2.2.2. It must be stressed that the functors $R_{n}, L_{n} ; \mathcal{M}_{n} \rightarrow \mathcal{F}$ are not exact. The simplest example of this is given by taking $\mathbb{F}=\mathbb{F}_{2}$ and studying the $\mathcal{M}_{2}$-module $S^{2}\left(\mathbb{F}^{2}\right)$, where $S^{2}$ is the second symmetric power. This module decomposes via the short exact sequence $\Lambda^{2}\left(\mathbb{F}^{2}\right) \rightarrow S^{2}\left(\mathbb{F}^{2}\right) \rightarrow \Lambda^{1}\left(\mathbb{F}^{2}\right)$, which is a non-trivial extension.

It is not difficult to show that $R_{2}\left(S^{2}\left(\mathbb{F}^{2}\right)\right) \cong \bar{I}$, where $I_{\mathbb{F}} \cong \mathbb{F} \oplus \bar{I}$ is the complete direct sum decomposition of $I_{\mathbb{F}}$. Moreover, $R_{2}\left(\Lambda^{1}\left(\mathbb{F}^{2}\right)\right) \cong \Lambda^{1}$. However, $R_{2}\left(\Lambda^{2}\left(\mathbb{F}^{2}\right)\right)$ is not isomorphic to $\bar{I} / \Lambda^{1}$, as is shown in Example 3.1.2.

2.3. Review of the simple functors and indecomposable injectives. This section recalls briefly the representation theory of $G L_{n}$ which underlies this paper, together with its connection with the category $\mathcal{F}$. A $p$-regular partition of length $s$ is taken to be a sequence of integers $\lambda=\left(\lambda_{1} \geq \lambda_{2} \geq \ldots \geq \lambda_{s}>0\right)$, where $\lambda_{i}>\lambda_{i+p}$, wherever this makes sense. If $\mathbb{F}$ is a prime field of characteristic $p$, the simple $G L_{n}$-modules are indexed by the $p$-regular partitions with $\lambda_{1}=n$. The simple module $S_{\lambda}$ indexed by $\lambda$ is the unique top composition factor of the Weyl module $W_{\lambda} \subset \Lambda^{\lambda}=\Lambda^{\lambda_{1}} \otimes \ldots \otimes \Lambda^{\lambda_{s}}$, where $\Lambda^{k}$ denotes the $k^{t h}$ exterior power of the natural representation of $G L_{n}$.

The simple functors in $\mathcal{F}$ are indexed over all $p$-regular partitions, $\lambda$; if $\lambda_{1}=n$, then the simple functor $F_{\lambda}$ is defined as the image of the natural map $L_{n} S_{\lambda} \rightarrow$ $R_{n} S_{\lambda}$, which is adjoint to the inverse of the unit of the $\left(L_{n}, E_{n}\right)$-adjunction (which is an isomorphism, since this is a recollement situtation). The simple functors $F_{\lambda}$ are self-dual under the operator $D: \mathcal{F} \rightarrow \mathcal{F}^{o p}$, namely $D F_{\lambda} \cong F_{\lambda}$ as functors, which corresponds to a similar statement for the simple $G L_{n}$-modules.

The indecomposable injectives in $\mathcal{F}$ are therefore indexed by the $p$-regular partitions. The injective envelope of the functor $F_{\lambda}$ is written as $I_{\lambda}$. Recall that $\operatorname{End}_{\mathcal{F}}\left(I_{V}\right) \cong \mathbb{F}[$ End $V]$, so that, if $\operatorname{dim} V=n=\lambda_{1}, I_{\lambda} \cong e_{\lambda} I_{V}$, where $e_{\lambda}$ is some primitive idempotent in $\mathbb{F}[$ End $V]$. There is an associated primitive idempotent in $\mathbb{F}\left[G L_{n}\right], \eta_{\lambda}$, taken so that $\mathbb{F}\left[G L_{n}\right] \eta_{\lambda}$ is the projective cover of $S_{\lambda}$ (which is also an injective envelope of $S_{\lambda}$ ). The embedding $\mathbb{F}\left[G L_{n}\right] \rightarrow \mathbb{F}\left[M_{n}\right]$ allows one to regard 
$\eta_{\lambda}$ as an idempotent in $\mathbb{F}\left[M_{n}\right]$; it is no longer primitive but, up to conjugacy, it is expressible as a sum:

$$
\eta_{\lambda}=e_{\lambda}+e_{\bar{\lambda}}
$$

where $\bar{\lambda}$ is the $p$-regular partition $\lambda_{1}-1 \geq \ldots \geq \lambda_{s}-1 \geq 0$; this was established in [HK]. This decomposition of $\eta_{\lambda}$ corresponds to a direct sum decomposition:

$$
\eta_{\lambda} I_{V} \cong I_{\lambda} \oplus I_{\bar{\lambda}}
$$

The following functors are also of interest:

\section{Definition 2.3.1.}

1. Write $\widetilde{I_{V_{n}}}$ for the functor $k_{n-1} I_{V_{n}} \in \mathcal{F}^{n}$. This is the largest sub-functor of $I_{V_{n}}$ which is zero when evaluated on $\mathbb{F}^{n-1}$.

2. If $\lambda$ is a $p$-regular partition with $\lambda_{1}=n$, then set $\widetilde{I_{\lambda}}:=k_{n-1} I_{\lambda}$.

It is useful to have another characterization of the functors $\widetilde{I_{V_{n}}}$, which is given by using the functors $R_{n}$. Recall that $\mathbb{F}\left[G L_{n}\right]$ is regarded as an object in $\mathcal{M}_{n}$ via the algebra map $\mathbb{F}\left[M_{n}\right] \rightarrow \mathbb{F}\left[G L_{n}\right]$. The duality functor $D: \mathcal{F} \rightarrow \mathcal{F}^{o p}$ restricts to a duality functor $D: \mathcal{M}_{n} \rightarrow \mathcal{M}_{n}^{o p}$; there is a natural isomorphism in $\mathcal{M}_{n}$ :

$$
D \mathbb{F}\left[G L_{n}\right] \cong \mathbb{F}\left[G L_{n}\right] .
$$

To prove this, it is sufficient to look at the $G L_{n}$-action; $D \mathbb{F}\left[G L_{n}\right]$ is isomorphic to $\mathbb{F}^{G L_{n}}$ with the transpose action. The latter space has a basis given by the maps $\alpha_{g}$, for $g \in G L_{n}$, which are defined by $x \mapsto 0$ if $x \neq g$ and $g \mapsto 1$. $G L_{n}$ acts transitively on this basis (regarded as a set), hence $\mathbb{F}^{G L_{n}}$ is a free permutation module, thus is isomorphic to $\mathbb{F}\left[G L_{n}\right]$.

\section{Proposition 2.3.2.}

1. $\widetilde{I_{V_{n}}}$ is isomorphic to $R_{n}\left(\mathbb{F}\left[G L_{n}\right]\right)$.

2. $\operatorname{End}_{\mathcal{F}}\left(\widetilde{I_{V_{n}}}\right) \cong \mathbb{F}\left[G L_{n}\right]$ as rings.

3. If $\lambda$ is a p-regular partition indexing a simple $G L_{n}$-module $S_{\lambda}$ and $P_{\lambda}$ is the projective cover of $S_{\lambda}$, then $\widetilde{I_{\lambda}} \cong R_{n}\left(P_{\lambda}\right)$.

Proof. The injective $I_{V_{n}}$ is isomorphic to $R_{n}\left(D \mathbb{F}\left[M_{n}\right]\right)$. The map $\mathbb{F}\left[M_{n}\right] \rightarrow \mathbb{F}\left[G L_{n}\right]$ in $\mathcal{M}_{n}$ dualizes to $\mathbb{F}\left[G L_{n}\right] \hookrightarrow D \mathbb{F}\left[M_{n}\right]$, which induces an injection $R_{n}\left(\mathbb{F}\left[G L_{n}\right]\right) \hookrightarrow$ $I_{V_{n}}$; hence it is sufficient to show that $R_{n} \mathbb{F}\left[G L_{n}\right]$ has the correct universal property.

It is clear that $R_{n} \mathbb{F}\left[G L_{n}\right] \in \mathcal{F}^{n}$, so consider $\operatorname{Hom}_{\mathcal{F}}\left(G, R_{n} \mathbb{F}\left[G L_{n}\right]\right)$, where $G \in$ $\mathcal{F}^{n}$. Using the adjunction $\left(E_{n}, R_{n}\right)$, this is isomorphic to $\operatorname{Hom}_{M_{n}}\left(G\left(\mathbb{F}^{n}\right), \mathbb{F}\left[G L_{n}\right]\right)$, which identifies by duality with $\operatorname{Hom}_{M_{n}}\left(\mathbb{F}\left[G L_{n}\right], D G\left(\mathbb{F}^{n}\right)\right)$. Since $D G\left(\mathbb{F}^{n}\right)$ necessarily belongs to $\mathcal{G} \mathcal{L}_{n} \subset \mathcal{M}_{n}$, this is isomorphic to $D G\left(\mathbb{F}^{n}\right)$, which establishes the required universal property.

In particular, this argument establishes an isomorphism of vector spaces

$$
\operatorname{End}_{\mathcal{F}}\left(\widetilde{I_{V_{n}}}\right) \cong \mathbb{F}\left[G L_{n}\right]
$$

There is an injection of rings $\mathbb{F}\left[G L_{n}\right] \hookrightarrow \operatorname{End}_{\mathcal{F}}\left(\widetilde{I_{V_{n}}}\right)$, given by restriction of the action of $\mathbb{F}\left[G L_{n}\right] \subset \mathbb{F}\left[M_{n}\right]$ on $I_{V_{n}}$. Hence, the above is an isomorphism of rings.

Finally, it is formal that $\widetilde{I_{V_{n}}}$ is injective in $\mathcal{F}^{n}$ and splits as a direct sum of functors $\widetilde{I_{\lambda}}$. This splitting is achieved by using primitive idempotents $\eta_{\lambda} \in \mathbb{F}\left[G L_{n}\right]$; then $P_{\lambda} \cong \mathbb{F}\left[G L_{n}\right] \eta_{\lambda}$ and $\widetilde{I_{\lambda}} \cong \eta_{\lambda} \widetilde{I_{V_{n}}}$. The stated isomorphism follows by standard arguments. 


\section{Co-WeYl OBJECTS AND $J$-GOOD FILTRATIONS}

The fundamental objects of study in this paper may now be defined:

Definition 3.0.1. Suppose that $\lambda$ is a $p$-regular partition with $\lambda_{1}=n$. The associated co-Weyl object $J_{\lambda}$ is defined to be $J_{\lambda}:=R_{n} S_{\lambda}$, where $R_{n}$ is the functor $\mathcal{G} \mathcal{L}_{n} \rightarrow \mathcal{F}^{n} \subset \mathcal{F}$.

It should be observed immediately that $J_{\lambda}$ is an analytic functor, with simple socle $F_{\lambda}$ and that $J_{\lambda}$ embeds in $I_{V_{n}}$. This may be seen by considering the inclusion $S_{\lambda} \hookrightarrow \mathbb{F}\left[G L_{n}\right]$ of $G L_{n}$-modules, which yields an injection $J_{\lambda}=R_{n} S_{\lambda} \hookrightarrow$ $R_{n}\left(\mathbb{F}\left[G L_{n}\right]\right) \cong \widetilde{I_{V_{n}}}$. As $\widetilde{I_{V_{n}}}$ is a sub-functor of $I_{V_{n}}$, this shows that $J_{\lambda}$ embeds in $I_{V_{n}}$. Moreover, by definition $E_{n} J_{\lambda} \cong S_{\lambda}$; it follows that $J_{\lambda}$ has a simple socle $F_{\lambda}$, since the factors of the socle of $I_{V_{n}}$ are detected by $E_{n}$.

The definition of the functor $R_{n}$ then ensures that $J_{\lambda}$ may be characterized up to isomorphism as being the largest sub-functor $F$ of $I_{V_{n}}$ such that $E_{n} F \cong S_{\lambda}$. (The last property implies that $E_{n-1} F=0$.) The following should be noted:

\section{Lemma 3.0.2.}

1. If $\lambda$ is a p-regular partition with $\lambda_{1}=n$, then $J_{\lambda} \cong R_{k} E_{k} J_{\lambda}$, for any $k \geq n$.

2. $J_{\lambda} \cong \operatorname{Hom}_{G L_{n}}\left(S_{\lambda}, \widetilde{I_{V_{n}}}\right)$.

Example 3.0.3. There are two basic examples of co-Weyl functors with which one should be familiar, working over the field $\mathbb{F}=\mathbb{F}_{2}$; we consider those induced from simple $G L_{n}$-modules, for some $n$.

1. By choice of indexing $S_{(n)} \cong \Lambda^{n}\left(\mathbb{F}^{n}\right) \cong \mathbb{F}$ is the trivial $G L_{n}$-module. In this case $J_{(n)}$ will be denoted by $\bar{D}(n)$; this is due to a relation with the Dickson invariants; namely $\bar{D}(n)$ may be seen to be isomorphic to the functor $\left(\widetilde{I_{V_{n}}}\right) G L_{n}$, where $G L_{n}$ acts naturally as endomorphisms of $\widetilde{I_{V_{n}}}$.

2. Write $\langle n\rangle$ for the 'triangular partition' $n>n-1>\ldots>1>0$ of length $n$. The module $S_{\langle n\rangle}$ is the Steinberg module for $G L_{n}$ and is (up to isomorphism) the only simple module which is projective. The corresponding functor $J_{\langle n\rangle}$ is usually written as $L(n)$ and is an injective functor. (The notation derives from a length filtration of certain modules over the Steenrod algebra, which motivated its use in topology [MP]). This is (up to isomorphism) the unique co-Weyl functor induced from a simple $G L_{n}$-module which is injective.

One should note that, for $n=1$, there are isomorphisms $L(1) \cong \bar{I} \cong \bar{D}(1)$.

One could also choose to study the 'Weyl objects' $D J_{\lambda}$ which are the duals of the co-Weyl objects. These are isomorphic to $L_{n} S_{\lambda}$, for suitable $n$. However, these functors are never analytic, for $n>0$; one should be aware that a choice has been made here to work with the category of analytic functors.

3.1. Non $\tilde{\nabla}$-nilpotence of $J_{\lambda}$. An important initial step in the arguments of this paper is given by using the functors $\tilde{\nabla}_{k}$ to give an approximation to the size of the functors $J_{\lambda}$, for varying $\lambda_{1}$. It is assumed throughout this section that $\mathbb{F}=\mathbb{F}_{2}$.

Recall from $[\mathrm{P}]$ that a functor is said to be $\tilde{\nabla}_{k}$-nilpotent if there exists some integer $N$ so that $\left(\tilde{\nabla}_{k}\right)^{N} F=0$; the full sub-category of $\tilde{\nabla}_{k}$-nilpotent functors is thick $[\mathrm{P}]$. Now, $\tilde{\nabla}_{k} I_{V_{n}}=0$ if $k>n$ (by the work of $[\mathrm{P}]$ ), so that it follows that $\tilde{\nabla}_{k} J_{\lambda}=0$ for $k>\lambda_{1}$, since $\tilde{\nabla}_{k}$ preserves injections. The key result is:

Proposition 3.1.1. The functor $J_{\lambda}$, with $\lambda_{1}=n$, is not $\tilde{\nabla}_{n}$-nilpotent. 
Proof. This relies upon studying the auxiliary functor $\mathcal{J}_{\lambda}$, which is defined by setting

$$
\mathcal{J}_{\lambda}:=\operatorname{Hom}_{G L_{n}}\left(S_{\lambda}, I_{V_{n}}\right) \text {. }
$$

The surjection $P_{\lambda} \rightarrow S_{\lambda}$ induces an inclusion $\mathcal{J}_{\lambda} \hookrightarrow I_{\lambda} \oplus I_{\bar{\lambda}} \cong \eta_{\lambda} I_{V_{n}}$ (see Section2).

There are non-trivial $G L_{n}$-maps $\mathbb{F}\left[G L_{n}\right] \rightarrow S_{\lambda} \hookrightarrow \mathbb{F}\left[G L_{n}\right]$ which induce maps $I_{V_{n}} \rightarrow \mathcal{J}_{\lambda} \rightarrow I_{V_{n}}$. The composite $\sigma$ is non-trivial and is induced by an element of the group ring $\mathbb{F}\left[G L_{n}\right]$, regarded as a sub-ring of $\mathbb{F}\left[M_{n}\right] \cong \operatorname{End}\left(I_{V_{n}}\right)$. Theorem 2.1.2 shows that $\tilde{\nabla}_{n} \sigma=\sigma$, so that $\left(\tilde{\nabla}_{n}\right)^{t} \sigma$ is non-zero for all $t>0$. It follows that $\left(\tilde{\nabla}_{n}\right)^{t} \mathcal{J}_{\lambda}$ is non-zero for all $t>0$, so that $\mathcal{J}_{\lambda}$ is not $\tilde{\nabla}_{n}$-nilpotent.

There is an exact sequence of functors with $G L_{n}$-action:

$$
0 \rightarrow \widetilde{I_{V_{n}}} \rightarrow I_{V_{n}} \rightarrow I_{V_{n-1}} \otimes \mathbb{F}^{\mathrm{hom}\left(\mathbb{F}^{n}, V_{n-1}\right)}
$$

where the second map is induced by composition

$$
\operatorname{hom}\left(\mathbb{F}^{n}, V_{n-1}\right) \times \operatorname{hom}\left(V_{n-1},-\right) \rightarrow \operatorname{hom}\left(\mathbb{F}^{n},-\right) .
$$

Applying the functor $\operatorname{Hom}_{G L_{n}}\left(S_{\lambda},-\right)$ to the exact sequence yields an exact sequence:

$$
0 \rightarrow J_{\lambda} \rightarrow \mathcal{J}_{\lambda} \rightarrow Q \rightarrow 0
$$

where $Q$ is a sub-functor of a finite direct sum of functors $I_{V_{n-1}}$. Thus, $J_{\lambda}$ is a sub-functor of $\mathcal{J}_{\lambda}$ and the cokernel $\mathcal{J}_{\lambda} / J_{\lambda}$ is $\tilde{\nabla}_{n}$-nilpotent. (More precisely, the cokernel is zero under $\tilde{\nabla}_{n}$.)

The category of $\tilde{\nabla}_{n}$-nilpotent functors is shown to be thick in $[\mathrm{P}]$. Thus, the short exact sequence 1 , in which $Q$ is $\tilde{\nabla}_{n}$-nilpotent and $\mathcal{J}_{\lambda}$ is not $\tilde{\nabla}_{n}$-nilpotent shows that $J_{\lambda}$ is not $\tilde{\nabla}_{n}$-nilpotent.

Example 3.1.2. As an immediate application of this result, consider the functor $\bar{D}(2)=R_{2}\left(\Lambda^{2}\left(\mathbb{F}^{2}\right)\right)$ which arises in Remark 2.2.2. The proposition shows that $\bar{D}(2)$ is not $\tilde{\nabla}_{2}$-nilpotent; this proves the claimed non-exactness, since the functor $\bar{I} / \Lambda^{1}$ is a quotient of $I_{\mathbb{F}}$ and is therefore $\tilde{\nabla}_{2}$-nilpotent, since $\tilde{\nabla}_{2}$ preserves surjections and $\tilde{\nabla}_{2} I_{\mathbb{F}}=0$.

Similarly, one has the following:

Corollary 3.1.3. Suppose that $\lambda$ is a p-regular partition with $\lambda_{1}=n$. The co-Weyl functor $J_{\lambda}$ is not a sub-quotient of a finite direct sum $\bigoplus_{\text {finite }} I_{V_{k}}$ with $k<n$.

Proof. The functor $\tilde{\nabla}_{n}$ preserves injections and surjections; since $\tilde{\nabla}_{n} J_{\lambda} \neq 0$ and $\tilde{\nabla}_{n}\left(\bigoplus_{\text {finite }} I_{V_{k}}\right)=0$, the result follows.

3.2. J-good filtrations. The material of this section is analogous to the notions used in the study of highest weight categories, as introduced by Cline, Parhsall and Scott. A brief review of this material is contained in Chapter 4 of $[\mathrm{M}]$. The concept of a $J$-good filtration corresponds to that of a $\nabla$-good filtration (for a different $\nabla$ !) in the setting of highest weight categories. Alternatively, this may be related to the study of good filtrations in algebraic group theory, as discussed in Section 4 of [J, Part II].

Definition 3.2.1. A functor $F$ is $J$-good if it admits a finite filtration $0=F_{0} \subset$ $F_{1} \subset \ldots \subset F_{N}=F$ such that each $F_{k} / F_{k-1}$ is non-zero and isomorphic to some coWeyl functor $J_{\lambda(k)}$. The integer $N$ is termed the $J$-length of $F$ and such a filtration is said to be $J$-good. 
There is an evident notion of a $D J$-good functor; namely $G$ is $D J$-good if it takes finite dimensional values and $D G$ is $J$-good.

A $J$-good functor $F$ is said to be $J^{n}$-good if it is isomorphic to $R_{n} M$, where $M$ is a $G L_{n}$-module and $R_{n}$ is the functor $R_{n}: \mathcal{G} \mathcal{L}_{n} \rightarrow \mathcal{F}^{n} \subset \mathcal{F}$. The full sub-category of $J^{n}$-good functors is denoted here by $\mathcal{J}^{n}$. The functor $R_{n}: \mathcal{G} \mathcal{L}_{n} \rightarrow \mathcal{F}^{n}$ factors through $\mathcal{J}^{n}$, since $\left.R_{n}\right|_{\mathcal{G} \mathcal{L}_{n}}$ is exact; in fact one has:

Proposition 3.2.2. The functors $E_{n}: \mathcal{J}^{n} \rightleftharpoons \mathcal{G} \mathcal{L}_{n}: R_{n}$ induce an equivalence of categories.

Proof. This is clear at the level of objects. For the morphisms, use the adjunction isomorphism and the isomorphism $E_{n} R_{n} M \cong M$ to deduce that $\operatorname{Hom}_{\mathcal{F}}\left(R_{n} M, R_{n} N\right)$ $\cong \operatorname{Hom}_{G L_{n}}\left(E_{n} R_{n} M, N\right) \cong \operatorname{Hom}_{G L_{n}}(M, N)$.

The defining property of the functors $J_{\lambda}$ establishes the following result:

Lemma 3.2.3. Suppose that $\mu, \lambda$ are $p$-regular partitions, with $\lambda_{1}=n$. If $F$ is an analytic functor with $E_{n}(F)=0$, then $\operatorname{Ext}_{\mathcal{F}}^{1}\left(F, J_{\lambda}\right)=0$. In particular, if $\mu_{1}>\lambda_{1}$, then $\operatorname{Ext}_{\mathcal{F}}^{1}\left(J_{\mu}, J_{\lambda}\right)=0$.

This lemma allows one to re-order any $J$-good filtration so that the largest coWeyl objects are at the bottom; recall that $k_{n}: \mathcal{F} \rightarrow \mathcal{F}^{n+1}$ is the right adjoint to the inclusion $\mathcal{F}^{n+1} \hookrightarrow \mathcal{F}$. If $F$ is an analytic functor with a finite socle, there exists $t$ so that $k_{t}(F)=0$. In this case, one may form the following natural filtration:

$$
0=k_{t}(F) \subset k_{t-1} F \subset \ldots \subset k_{0} F \subset F .
$$

Combining Lemma 3.2.3 with Proposition 2.2.1, one may conclude the following result:

Proposition 3.2.4. An analytic functor $F$ with finite socle is $J$-good if and only if each functor $k_{s} F / k_{s+1} F$ in the filtration (2) is $J$-good. This holds if and only if $k_{s} F / k_{s+1} F \cong R_{s+1} M$ for some $G L_{s+1}$-module $M$.

There is another related result:

Proposition 3.2.5. An analytic functor $F$ with finite socle is $J$-good if and only if one of the following conditions hold:

1. $E_{s-1} F=0$, for some $s>0$, the functor $k_{s} F$ is $J$-good and the sequence

$$
k_{s} F \rightarrow F \rightarrow R_{s} E_{s} F
$$

is short exact.

2. For all $s$, the unit $F \rightarrow R_{s} E_{s} F$ is surjective.

Proof. The first statement is clear: if $E_{s-1} F=0$, then $E_{s} F$ should be regarded as an object in $\mathcal{G} \mathcal{L}_{s} \subset \mathcal{M}_{s}$. Since $\left.R_{s}\right|_{\mathcal{G} \mathcal{L}_{s}}$ is an exact functor, it follows that $R_{s} E_{s} F$ is $J^{s}$-good. By hypothesis, $k_{s} F$ is $J$-good, so that the result follows.

Consider the second statement; suppose that $F$ is an analytic functor with finite socle and that the unit maps $F \rightarrow R_{s} E_{s} F$ are surjective, for every $s$. By the first part of the proposition, it will suffice to show that the maps $k_{t} F \rightarrow R_{t+1} E_{t+1} k_{t} F$ are surjective for every $t$, since this will allow a decreasing induction on $t$.

To prove this, form the commutative diagram:

$$
\begin{array}{ccccc}
k_{t} F & \rightarrow & F & \rightarrow & R_{t} E_{t} F \\
\downarrow & & \downarrow & & \downarrow \\
0 \rightarrow R_{t+1} E_{t+1} k_{t} F & \rightarrow & R_{t+1} E_{t+1} F & \rightarrow & R_{t+1} E_{t+1} R_{t} E_{t} F
\end{array}
$$


The top row is a short exact sequence, by the hypothesis and the definition of the functor $k_{t}$, whereas the bottom row is exact (not necessarily short exact), by the left exactness of $R_{t+1}$. The vertical arrows are the units of the $\left(E_{t+1}, R_{t+1}\right)$-adjunction.

Observe that $R_{t+1} E_{t+1} R_{t} E_{t} F$ is naturally isomorphic to $R_{t} E_{t} F$, so that the unit map $\iota$ is the identity, up to isomorphism. Hence, the bottom row is a short exact sequence. Now, the hypothesis shows that $F \rightarrow R_{t+1} E_{t+1} F$ is a surjection; since $\iota$ is an isomorphism, an easy diagram chase shows that the map $k_{t} F \rightarrow R_{t+1} E_{t+1} k_{t} F$ is surjective. This completes the proof that $F$ is $J$-good.

It is left as an exercise to show that, if $F$ is a $J$-good functor, then all the natural maps $F \rightarrow R_{s} E_{s} F$ are surjective.

The basic facts about $J$-good functors are summarized as:

\section{Theorem 3.2.6.}

1. Suppose that $F \rightarrow G \rightarrow H$ is a short exact sequence of analytic functors; if any two of the functors are $J$-good, then so is the third.

2. If $F \oplus H$ is a J-good functor, then so are both $F$ and $H$.

3. If $F, G$ are $J$-good sub-functors of an analytic functor $H$ with finite socle, then $F \cap G$ and $F+G$ are both $J$-good.

4. If $H$ is an analytic functor with finite socle, then there is a well-defined maximal $J$-good sub-functor $J(H) \hookrightarrow H$.

Proof. Part 1: It is clear that, if $F \rightarrow G \rightarrow H$ is a short exact sequence, with $F, H$ $J$-good functors, then $G$ is a $J$-good functor. Hence it remains to consider the case $F, G J$-good and the case $G, H J$-good.

Case 1: Suppose that $F, G$ are $J$-good and choose $t$ minimal so that $k_{t} G=0$. By the left exactness of $k_{t}$, it follows that $k_{t} F=0$. Now write $Q$ for the quotient $k_{t-1} G / k_{t-1} F$, which is $J^{t}$-good, by Proposition 3.2.4, since the functors $k_{t-1} G$, $k_{t-1} G$ are both $J^{t}$-good. The universal property of the cokernel induces a canonical map $Q \rightarrow H$; I claim that this map is an injection. The kernel $\operatorname{ker}\{Q \rightarrow H\}$ injects in $F / k_{t-1} F$, by standard arguments. By the definition of $k_{t-1} F, F / k_{t-1} F$ embeds in a finite direct sum $\bigoplus_{\text {finite }} I_{V_{t-1}}$; since $\operatorname{Hom}_{\mathcal{F}}\left(Q, I_{V_{t-1}}\right)$ is zero by construction, this implies that the kernel of $Q \rightarrow H$ is zero.

Now consider the commutative diagram, in which the rows and columns are short exact sequences:

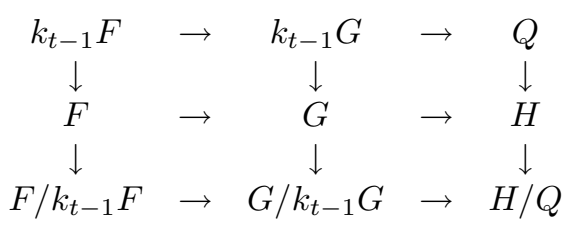

Now, $H$ is $J$-good if $H / Q$ is $J$-good, since $Q$ is $J$-good. This allows one to perform an induction upon the $J$-length of $G$, since the hypotheses ensure that $F / k_{t-1} F$ and $G / k_{t-1} G$ are both $J$-good and the $J$-length of $G / k_{t-1} G$ is less than that of $G$. The induction is started by the case $G=0$.

Case 2: $G, H$ both $J$-good. This result is proved by an induction upon the $J$-length of $G$, the initial case being $G=0$, which is trivial. Now choose $s$ so that $k_{s} G=0$ and $k_{s-1} G \neq 0$. It follows that $H$ embeds in a finite direct sum of $I_{V_{s}}$ 's, since $H$ is $J$-good and $J_{\lambda}$, for $\lambda_{1}>s$ cannot occur as a sub-quotient of $G$ (which embeds in a finite direct sum $\bigoplus_{\text {finite }} I_{V_{s}}$ ), by Proposition 3.2.4. 
For the inductive step, there is some $J_{\mu}$, with $\mu_{1}=s$, which embeds in $G$; by the defining properties of $J_{\mu}$ and the choice of $s$, the composite map $J_{\mu} \rightarrow G \rightarrow H$ is either injective or trivial.

In the first case, there is a short exact sequence $F \rightarrow G / J_{\mu} \rightarrow H / J_{\mu}$. In the second, the map $J_{\mu} \rightarrow G$ factors through $F$ and there is a short exact sequence $F / J_{\mu} \rightarrow G / J_{\mu} \rightarrow H$. In both cases, this forms the basis of the inductive step, since the $J$-length of $G / J_{\mu}$ is less than that of $G$. (Observe in the second case that $F$ is $J$-good if and only if $F / J_{\mu}$ is $J$-good.)

Statement 2 may be regarded as an application of Proposition 3.2.5, and is left as an exercise.

To prove the third statement, observe that there is a short exact sequence $F \cap$ $G \rightarrow F \oplus G \rightarrow F+G$. This means that we may consider $F \cap G$ as a sub-functor of $F \oplus G$; moreover, it suffices to show that $F \cap G$ is $J$-good.

This may be proved by an induction on the $J$-length of $F \oplus G$. Choose $t$ maximal so that $k_{t-1} F=0=k_{t-1} G$ and suppose (without loss of generality) that $k_{t} F \neq 0$. Now, there is some $\lambda$ with $\lambda_{1}=t$ so that $J_{\lambda}$ embeds in $F$, since $F$ is $J$-good.

By choice of $t$, any map $F_{\lambda} \hookrightarrow F$ extends uniquely to a map $J_{\lambda} \hookrightarrow F$ and the same fact holds for $G$. Suppose that the factor $F_{\lambda} \in \operatorname{soc} F$ lies in $F \cap G$, then $J_{\lambda}$ embeds in $F \cap G$. Consider the embedding $(F \cap G) / J_{\lambda} \hookrightarrow\left(F / J_{\lambda}\right) \oplus\left(G / J_{\lambda}\right)$ and conclude that $(F \cap G) / J_{\lambda}$ is $J$-good, by the induction on the $J$-length of the direct sum.

Otherwise, there is an embedding $\left(F / J_{\lambda}\right) \cap G \hookrightarrow\left(F / J_{\lambda}\right) \oplus G$ and the result again follows by an induction on the $J$-length of the direct sum.

The final statement is an application of the third statement; $J(F)$ is defined as the sum of all the $J$-good sub-functors of $F$. (It is not difficult to show that there are only finitely many $J$-good sub-functors of an analytic functor $F$ with a finite socle.)

\section{The indecomposable injectives in $\mathcal{F}$ ARE $J$-GOod}

The purpose of this section is to prove that the indecomposable injective functors in $\mathcal{F}$ are $J$-good. This has an important application to the Cartan matrices of $\mathbb{F}\left[M_{n}\right]$ and $\mathbb{F}\left[G L_{n}\right]$.

Theorem 4.0.1. The indecomposable injective functors in $\mathcal{F}$ are $J$-good.

The proof of the theorem given here relies on the following result:

Lemma 4.0.2. The natural map induced by composition,

$$
\operatorname{hom}\left(\mathbb{F}^{n}, V\right) \times_{\operatorname{End}\left(\mathbb{F}^{n}\right)} \operatorname{hom}\left(W, \mathbb{F}^{n}\right) \rightarrow \operatorname{hom}(W, V)
$$

is an injection. The image identifies with $\operatorname{hom}_{\leq n}(W, V)$, the set of morphisms of rank $\leq n$.

Proof. The proof is by induction upon $n$; the case $n=0$ is trivial; to carry out the inductive step, consider the set $S_{n}=\operatorname{hom}\left(\mathbb{F}^{n}, V\right) \times_{\operatorname{End}\left(\mathbb{F}^{n}\right)} \operatorname{hom}\left(W, \mathbb{F}^{n}\right)$ and fix an idempotent $e \in \operatorname{End}\left(\mathbb{F}^{n}\right)$, which is a projection onto $\mathbb{F}^{n-1}$. Suppose that 
$e$ factors as $\mathbb{F}^{n} \stackrel{p}{\rightarrow} \mathbb{F}^{n-1} \stackrel{i}{\hookrightarrow} \mathbb{F}^{n}$ and observe that there is a natural isomorphism $e \operatorname{End}\left(\mathbb{F}^{n}\right) e \cong \operatorname{End}\left(\mathbb{F}^{n-1}\right)$.

There is an inclusion $S_{n-1}=\operatorname{hom}\left(\mathbb{F}^{n-1}, V\right) \times_{\operatorname{End}\left(\mathbb{F}^{n-1}\right)} \operatorname{hom}\left(W, \mathbb{F}^{n-1}\right) \hookrightarrow S_{n}$ which is induced by the maps $p: \mathbb{F}^{n} \rightarrow \mathbb{F}^{n-1}$ and $i: \mathbb{F}^{n-1} \hookrightarrow \mathbb{F}^{n}$. I claim that the complement identifies (as a set) with $T:=\operatorname{inj}\left(\mathbb{F}^{n}, V\right) \times_{G L_{n}} \operatorname{surj}\left(W, \mathbb{F}^{n}\right)$, where $\operatorname{inj}(-,-)$ denotes the set of injections and $\operatorname{surj}(-,-)$ denotes the set of surjections. Here, $T$ is regarded as a sub-set of $S_{n}$ in the evident way.

To see this, suppose that $(\alpha, \beta) \in \operatorname{hom}\left(\mathbb{F}^{n}, V\right) \times \operatorname{hom}\left(W, \mathbb{F}^{n}\right)$ represents an element in $S_{n}$ which is not in $T$. Thus, either $\alpha$ is not injective or $\beta$ is not surjective. Suppose that $\alpha$ is not injective, then there exists $\gamma \in \operatorname{hom}\left(\mathbb{F}^{n}, V\right)$ and $r \in \operatorname{End}\left(\mathbb{F}^{n}\right)$ so that $\alpha=\gamma e r$. Hence, $(\alpha, \beta)$ is equivalent to $(\gamma e r, \beta) \sim(\gamma e, e r \beta)$, using the fact that $e^{2}=e$; thus $(\alpha, \beta)$ represents an element in $S_{n-1}$. A similar argument works if $\beta$ is not surjective.

Hence $S_{n}$ identifies with $S_{n-1} \amalg T$; by induction the composition map $S_{n-1} \rightarrow$ $\operatorname{hom}(W, V)$ is injective, whereas the composition map restricted to $T$ may easily be shown to be injective, by the uniqueness of the factorization of a map of rank $n$ through $\mathbb{F}^{n}$, up to elements of Aut $\left(\mathbb{F}^{n}\right)$. Conclude that the composition map $S_{n} \hookrightarrow \operatorname{hom}(W, V)$ is injective. Finally, it is clear that the image of this map is the set $\operatorname{hom}_{\leq n}(W, V)$ of maps of rank $\leq n$.

Proof of Theorem 4.0.1. To prove the result, it suffices to show that the functors $I_{V_{n}}$ are $J$-good for all $n$, by Theorem 3.2.6. This is achieved by using the criterion of Proposition 3.2.5. For typographical reasons, it is easiest to dualize and study the projective functors $P_{W}$, so that the criterion becomes:

- The functor $P_{W}$ is $D J$-good if and only if the maps $L_{n} E_{n} P_{W} \rightarrow P_{W}$ are injective for each $n \geq 0$.

One has $E_{n} P_{W} \cong \mathbb{F}\left[\operatorname{hom}\left(W, \mathbb{F}^{n}\right)\right]$, so that

$$
L_{n} E_{n} P_{W} \cong \mathbb{F}\left[\operatorname{hom}\left(\mathbb{F}^{n},-\right)\right] \otimes_{\operatorname{End}\left(\mathbb{F}^{n}\right)} \mathbb{F}\left[\operatorname{hom}\left(W, \mathbb{F}^{n}\right)\right] ;
$$

this is isomorphic to $\mathbb{F}\left[\operatorname{hom}\left(\mathbb{F}^{n},-\right) \times_{\operatorname{End}\left(\mathbb{F}^{n}\right)} \operatorname{hom}\left(W, \mathbb{F}^{n}\right)\right]$. The map $L_{n} E_{n} P_{W} \rightarrow P_{W}$ identifies with the composition map.

Thus, one may appeal to the lemma above, which shows that the composition map $\operatorname{hom}\left(\mathbb{F}^{n}, V\right) \times_{\operatorname{End}\left(\mathbb{F}^{n}\right)} \operatorname{hom}\left(W, \mathbb{F}^{n}\right) \rightarrow \operatorname{hom}(W, V)$ is injective, for all $V$. Hence, the same is true after 'linearization' by $\mathbb{F}[-]$, so that the result is established. The map $L_{n} E_{n} P_{W} \rightarrow P_{W}$ identifies with the inclusion $\mathbb{F}\left[\operatorname{hom}_{\leq n}(W,-)\right] \rightarrow$ $\mathbb{F}[\operatorname{hom}(W,-)]$.

Remark 4.0.3. Essentially, one is analysing the filtration of $P_{W}$ given by the subfunctors $\mathbb{F}\left[\operatorname{hom}_{\leq n}(W,-)\right]$; a similar analysis appears in [K4]. It is more direct to prove the theorem by showing that the quotients

$$
\mathbb{F}\left[\operatorname{hom}_{\leq n}(W, V)\right] / \mathbb{F}\left[\operatorname{hom}_{\leq n-1}(V, W)\right]
$$

are $J$-good.

Notation 4.0.4. Write $\sharp \mu(F)$ for the dimension $\operatorname{dim} \operatorname{Hom}_{\mathcal{F}}\left(F, I_{\mu}\right)$, which is equal to the number of composition factors of $F_{\mu}$ in $F$. The partitions may be ordered (for example by a lexicographical ordering) so that $\lambda<\mu$ if $\lambda_{1}<\mu_{1}$. Then one may consider the following matrices of coefficients:

1. $\sharp \mu\left(I_{\lambda}\right)$ : the Cartan matrix for the category $\mathcal{F}$. 
2. $\sharp \mu\left(J_{\nu}\right)$ : the multiplicity matrix for the functor $J_{\nu}$.

3. $\left(\alpha_{\lambda \nu}\right)$ : the $J$-good decomposition matrix, which is defined by the equation:

$$
I_{\lambda}=\alpha_{\lambda \nu} J_{\nu}
$$

which indicates that $I_{\lambda}$ has a $J$-good filtration in which $J_{\lambda}$ occurs with multiplicity $\alpha_{\lambda \nu}$.

The defining properties of the co-Weyl objects mean that the matrices $\sharp \mu\left(J_{\nu}\right)$ and $\left(\alpha_{\lambda \nu}\right)$ have special forms. The matrix $\sharp \mu\left(J_{\nu}\right)$ is triangular, with 1's on the diagonal. Namely:

$$
\sharp \mu\left(J_{\nu}\right)= \begin{cases}0 & \text { if } \mu_{1} \leq \nu_{1} \text { and } \mu \neq \nu, \\ 1 & \text { if } \mu=\nu .\end{cases}
$$

The reader is encouraged to write down the block form of this matrix.

Similarly, Proposition 3.2.4 implies that $\alpha_{\lambda \nu}=0$ if $\nu_{1}>\lambda_{1}$. Moreover, the coefficients $\alpha_{\lambda \nu}$ for $\lambda_{1}=\nu_{1}=n$ identify with the Cartan matrix $C_{\lambda \nu}$ for the finite group algebra $\mathbb{F}\left[G L_{n}\right]$, by the same result. Again, the reader is encouraged to picture the block form of this matrix.

These matrices are related by the equation:

$$
\sharp \mu\left(I_{\lambda}\right)=\alpha_{\lambda \nu} \sharp \mu\left(J_{\nu}\right)
$$

in which a summation over $\nu$ is implicit.

This equation shows that the matrix $\sharp \mu\left(I_{\lambda}\right)$ is determined by the other two matrices; an important fact is that the converse is true:

Proposition 4.0.5. The Cartan invariants $\sharp \mu\left(I_{\lambda}\right)$ determine the matrices $\sharp \mu\left(J_{\nu}\right)$ and $\left(\alpha_{\lambda \nu}\right)$.

Proof. Prove by induction on $n$ that:

Hypothesis stage $n$ : The matrix $\sharp \mu\left(I_{\lambda}\right)$ determines $\left(\alpha_{\lambda \nu}\right)$ for $\lambda_{1}, \nu_{1} \leq n$ and $\sharp \mu\left(J_{\nu}\right)$ for $\nu_{1}<n$.

The induction starts at $n=0$, where the statement is trivial to establish. For the inductive step, suppose that the hypothesis holds for $n$ and start by determining the coefficients $\sharp \mu\left(J_{\nu}\right)$ for $\nu_{1}=n$. Equation (3), when $\lambda_{1}=n$, may be rewritten as

$$
\sum_{\nu_{1}=n} C_{\lambda \nu} \sharp \mu\left(J_{\nu}\right)=\sharp \mu\left(I_{\lambda}\right)-\sum_{\kappa_{1}<n} \alpha_{\lambda \kappa} \sharp \mu\left(J_{\kappa}\right),
$$

where $C_{\lambda \nu}=\alpha_{\lambda \nu}$ for $\nu_{1}=n$ identifies with the Cartan matrix for $\mathbb{F}\left[G L_{n}\right]$, which is invertible [CR, Theorem 18.25]. The inductive hypothesis implies that the coefficients $C_{\lambda \nu}$ and all terms on the right hand side are known, hence this equation determines the coefficients $\sharp \mu\left(J_{\nu}\right)$.

Now determine the coefficients $\left(\alpha_{\lambda \nu}\right)$ for $\lambda_{1}, \nu_{1} \leq n+1$ as follows. The coefficients $\sharp \mu\left(J_{\nu}\right)$ are known for $\mu_{1}, \nu_{1} \leq n+1$, by the special form of this matrix, which has blocks which are identity matrices on the diagonal. Since the matrix is triangular, it is also invertible; hence, the matrix equation

$$
\sharp \mu\left(I_{\lambda}\right)=\alpha_{\lambda \nu} \sharp \mu\left(J_{\nu}\right)
$$

determines $\left(\alpha_{\lambda \mu}\right)$ in the given range. 
This completes the proof of the inductive step.

It is worth noting that the above result has established the corollary below.

Corollary 4.0.6. The Cartan matrix for $\mathbb{F}\left[M_{n}\right]$ determines the Cartan matrix for $\mathbb{F}\left[G L_{n}\right]$.

4.1. Example of a $J$-good filtration. We may consider the Grothendieck group of $J$-good functors; namely, form the free abelian group $(G J)_{0} \mathcal{F}$, which is generated by symbols $\left[J_{\lambda}\right]$, where $\lambda$ ranges over all $p$-regular partitions. If $G$ is a $J$-good functor, then write $[G]$ for the element of $(G J)_{0} \mathcal{F}$ which is given inductively as follows: if $F \rightarrow G \rightarrow H$ is a short exact sequence of $J$-good functors, then set $[G]=[F]+[H]$. The results of Section 5 imply that this may be given a ring structure by the tensor product of functors.

Working in $(G J)_{0} \mathcal{F}$, when $\mathbb{F}=\mathbb{F}_{2}$, the above is illustrated by the study of $I_{V_{3}}$. Recall that the simple functors $F_{\lambda}$, with $\lambda_{1} \leq 3$, are the following:

$$
\begin{array}{ll}
F_{(0)} \cong \mathbb{F}, & \\
F_{(1)} \cong \Lambda^{1}, F_{(2)} \cong \Lambda^{2}, F_{(3)} \cong \Lambda^{3} & \text { (the exterior power functors), } \\
F_{(2,1)}, F_{(3,2,1)} & \text { (functors induced from Steinberg modules), } \\
F_{(3,2)}, F_{(3,1)} . &
\end{array}
$$

The work of [PS] shows that $I_{V_{2}}$ decomposes as

$$
I_{V_{2}} \cong \mathbb{F} \oplus I_{(2)} \oplus I_{(2,1)}^{\oplus 2} \oplus I_{(1)}^{\oplus 2} .
$$

$I_{(2,1)}$ is isomorphic to the co-Weyl module $J_{(2,1)}$, which is usually written as $L(2)$, whereas $I_{(1)}$ is usually written as $\bar{I}$. There is a decomposition of $I_{(2)}$ as $\left[I_{(2)}\right]=$ $2[\bar{D}(2)]+[\bar{I}]$. To calculate the $J$-good filtration of $I_{V_{3}}$, one uses the inductive method of Corollary 4.0.6, starting with the following Cartan matrix for $\mathbb{F}\left[M_{3}\right]$ (Table 1), which was taken from [HHS, Theorem 4.2].

TABLE 1

\begin{tabular}{|l|cccccccc|}
\hline & $(0)$ & $(1)$ & $(2,1)$ & $(2)$ & $(3,2,1)$ & $(3,2)$ & $(3,1)$ & $(3)$ \\
\hline$(0)$ & 1 & & & & & & & \\
$(1)$ & & 1 & & 1 & & & & 1 \\
$(2,1)$ & & & 1 & & & 1 & 1 & \\
$(2)$ & & 1 & & 3 & & & 2 & 3 \\
$(3,2,1)$ & & & & & 1 & & & \\
$(3,2)$ & & & 1 & & & 4 & 3 & 1 \\
$(3,1)$ & & & 1 & 2 & & 3 & 6 & 3 \\
$(3)$ & & 1 & & 3 & & 1 & 3 & 5 \\
\hline
\end{tabular}

Now, the algorithm may be applied- the details are left to the reader as an exercise; one calculates the results displayed in Table 2, in which the boxed terms correspond to the Cartan matrix for $\mathbb{F}\left[G L_{3}\right]$.

4.2. Ext groups for $J^{t}$-good functors. Using the fact that the injective indecomposable functors in $\mathcal{F}$ are $J$-good, we may now describe Ext groups between $J^{t}$-good functors. 
TABLE 2

\begin{tabular}{|l|ccc|cccc|}
\hline & $J_{(1)}$ & $J_{(2,1)}$ & $J_{(2)}$ & $J_{(3,2,1)}$ & $J_{(3,2)}$ & $J_{(3,1)}$ & $J_{(3)}$ \\
\hline & & & & & & & \\
\cline { 5 - 8 }$I_{(3,2,1)}$ & & & & 1 & & & \\
$I_{(3,2)}$ & & 1 & & & 3 & 2 & 1 \\
$I_{(3,1)}$ & & 1 & 2 & & 2 & 3 & 1 \\
$I_{(3)}$ & 1 & & 2 & & 1 & 1 & 2 \\
\hline
\end{tabular}

Lemma 4.2.1. Suppose that $F \rightarrow G$ is a surjection between $J$-good functors and that $F \hookrightarrow R_{t} E_{t} F$ is an embedding, then the map $k_{t-1} F \rightarrow k_{t-1} G$ is a surjection.

Proof. The hypothesis ensures that $G$ embeds in $R_{t} E_{t} G$, since the $J$-factors of $G$ are $J$-factors of $F$ and the largest $J$-factors always occur at the bottom of a $J$-good filtration. Reduce to the case that $k_{t-1} F=0$, by observing that $k_{t-1} F, k_{t-1} G$ are both $J^{t}$-good functors, so that the image $H$ of the map $k_{t-1} F \rightarrow k_{t-1} G$ is $J$-good; thus one can reduce to studying the induced surjection $F / k_{t-1} F \rightarrow G / H$. Now, $F / k_{t-1} F$ embeds in $\bigoplus_{\text {finite }} I_{V_{t-1}}$; conclude by Corollary 3.1.3 that $G / H$ does not contain a sub-functor $J_{\mu}$, with $\mu_{1}=t$. It follows immediately that $H=k_{t-1} G$, establishing the result.

Proposition 4.2.2. Suppose that $F, G$ are $J^{t}$-good functors, isomorphic respectively to $R_{t} M$ and $R_{t} N$, for $M, N \in \mathcal{G L}_{t}$. Then $\operatorname{Ext}_{\mathcal{F}}^{*}(F, G) \cong \operatorname{Ext}_{G L_{t}}^{*}(M, N)$.

Proof. By Proposition 3.2.2, it is sufficient to show that there is an isomorphism: $\operatorname{Ext}_{\mathcal{F}}^{*}(F, G) \cong \operatorname{Ext}_{\mathcal{J}^{t}}^{*}(F, G)$. Consider a minimal injective resolution of $G$ in $\mathcal{F}$, $0 \rightarrow G \rightarrow I^{\bullet}$. It is not difficult to show that each $I^{k}$ embeds in a finite direct sum $\bigoplus I_{V_{t}}$. Moreover, since $k_{t-1}$ is a right adjoint, $k_{t-1} I^{\bullet}$ is a complex of injectives in $\mathcal{F}^{t}$. Since $\mathcal{J}^{t}$ is a full sub-category of $\mathcal{F}^{t}$, it suffices to show that the complex $0 \rightarrow G \rightarrow k_{t-1} I^{\bullet}$ is exact.

This is established by using the fact that each functor $I^{k}$ is $J$-good. The injective resolution breaks into a Yoneda product of short exact sequences $Z_{n} \rightarrow I^{n} \rightarrow Z_{n+1}$, in which the functors $Z_{n}$ are $J$-good, since the category of $J$-good functors is thick. The lemma applies to show that the sequences $k_{t-1} Z_{n} \rightarrow k_{t-1} I^{n} \rightarrow k_{t-1} Z_{n+1}$ are all short exact.

\section{A homological Characterization of $J$-GOOD FUnCtors}

The purpose of this section is to give a homological characterization of a $J$-good functor. The main result is:

Theorem 5.0.1. An analytic functor $F$ with finite socle is $J$-good if and only if it admits an injective resolution $0 \rightarrow F \rightarrow I^{\bullet}$ such that there exists an integer $d$ so that each $I^{k}$ embeds in a finite direct sum $\bigoplus_{\text {finite }} I_{V_{d}}$.

Suppose that $F$ is an analytic functor with a finite socle; there is a minimal $N$ so that $F$ embeds in $R_{N} E_{N} F$ (equivalently, $k_{N} F=0$ ). Write this $N$ as emb $F$, standing for embedding dimension of $F$. It is clear that emb $F \leq N$ if and only if $F$ embeds in a finite direct sum $\bigoplus_{\text {finite }} I_{V_{N}}$.

By Theorem 3.2.6, there is a well-defined notion of a maximal $J$-good functor $J(F)$ of an analytic functor $F$ with finite socle. This allows one to take the following definition: 
Definition 5.0.2. If $F$ is not a $J$-good functor, then write $d_{J}(F):=\operatorname{emb} F / J(F)$.

The proof of the main result of this section uses the interplay between $d_{J}(F)$ and emb $F$ in injective resolutions of functors. The crucial step is the following:

Lemma 5.0.3. Suppose that $F$ is an analytic functor with finite socle and $I_{F}$ is the injective envelope of $F$. If $F$ is not $J$-good, then $d_{J}\left(I_{F} / F\right)>d_{J}(F)$.

Proof. Consider the embedding $F \hookrightarrow I_{F}$; by hypothesis, the functor $F$ is not $J$ good, so that $d_{J}(F)=n$, for some $n>0$. Now, using the fact that $I_{F}$ is $J$-good, we may consider the $J$-good functor $G=k_{n}\left(I_{F}\right)=\operatorname{ker}\left\{I_{F} \rightarrow R_{n} E_{n} I_{F}\right\}$. Take $\hat{F}$ to be the functor $\hat{F}:=F+G$. It is clear that $J(\hat{F})=G+J(F)$, so that it follows that $d_{J}(\hat{F})=d_{J}(F)$. Moreover, a similar reasoning shows that $d_{J}\left(I_{F} / \hat{F}\right)=d_{J}\left(I_{F} / F\right)$, using the choice of $n$.

Hence, it suffices to prove the result for $\hat{F}$. By construction, $I_{F} / G$ embeds in a finite direct sum $\bigoplus_{\text {finite }} I_{V_{n}}$. Moreover, by the choice of $n$, there is an embedding $J_{\lambda} \hookrightarrow I_{F} / G$, for some $\lambda$ with $\lambda_{1}=n$, so that the composite $J_{\lambda} \rightarrow I_{F} / G \rightarrow I_{F} / \hat{F}$ is non-trivial.

This implies, by the defining property of $J_{\lambda}$, that $\operatorname{soc}\left(I_{F} / \hat{F}\right)$ contains a composition factor $F_{\nu}$, with $\nu_{1}>n$, which lies in the image of the above map. Thus, to prove the result, it suffices to show that $J_{\nu}$ does not embed in $I_{F} / \hat{F}$. This follows by applying Corollary 3.1.3, since $I_{F} / \hat{F}$ is a quotient of $I_{F} / G$, which embeds in $\bigoplus_{\text {finite }} I_{V_{n}}$, so that $I_{F} / \hat{F}$ is a sub-quotient of $\bigoplus_{\text {finite }} I_{V_{n}}$.

Proof of Theorem 5.0.1. If $F$ is $J$-good, then $F$ embeds in a finite direct sum $I^{0}=\bigoplus_{\text {finite }} I_{W}$, where $\operatorname{dim}(W)=\operatorname{emb} F$. The functor $I^{0} / F$ is $J$-good, by Theorem 3.2.6, with finite socle and $\operatorname{emb}\left(I^{0} / F\right) \leq \operatorname{emb} F$. Thus, an inductive argument shows that, if $F$ is $J$-good, then $F$ has an injective resolution of the form given.

For the converse, suppose that $F$ is an analytic functor which admits an injective resolution of the form given; thus, we may assume that $F$ has a minimal injective resolution:

$$
0 \rightarrow F \rightarrow I^{0} \rightarrow I^{1} \rightarrow \ldots \rightarrow I^{k} \rightarrow \ldots,
$$

where each $I^{k}$ has finite socle and emb $I^{k} \leq d$, for some fixed $d$.

Write $Z_{n+1}$ for the image of $I^{n} \rightarrow I^{n+1}$, so that $I^{n+1}$ is the injective envelope of $Z_{n+1} \cong I^{n} / Z_{n}$. In particular, emb $I^{n+1}=\operatorname{emb}\left(I^{n} / Z_{n}\right)$.

Now, suppose that $F$ is not $J$-good; then, since there are short exact sequences $Z_{n} \rightarrow I^{n} \rightarrow Z_{n+1}$, Theorem 3.2.6 shows that each $Z_{n}$ is not $J$-good, since $I^{n}$ is $J$-good by Theorem 4.0.1. This means that Lemma 5.0.3 may be applied, showing that $d_{J}\left(I^{n} / Z_{n}\right)>d_{J}\left(Z_{n}\right)$. In particular, since $I^{n} / Z_{n} \cong Z_{n+1}$, this shows that $d_{J}\left(Z_{n}\right)$ is not bounded as $n$ increases.

Now, if $F$ is an analytic functor with finite socle, Proposition 3.2.4 implies that emb $F \geq d_{J}(F)$. Hence, the above argument shows that $\operatorname{emb}\left(Z_{n}\right)$ is not bounded. Since $I^{n}$ is the injective envelope of $Z_{n}$, it follows that emb $I^{n}$ is not bounded, which is a contradiction.

The form of the injective resolution of a $J$-good functor $F$ is determined by an injective resolution of $E_{d} F$ as an $M_{d}$-module, if $d$ is sufficiently large:

Proposition 5.0.4. Suppose that $F$ is a J-good functor with $\mathrm{emb} F=d$. If $n \geq d$ and $J^{\bullet}$ is an injective resolution of $E_{n} F$ in $\mathcal{M}_{n}$, then $R_{n} J^{\bullet}$ is an injective resolution of $F$. 
An important corollary to the theorem is that this allows one to show that certain constructions between $J$-good functors give $J$-good functors:

\section{Corollary 5.0.5.}

1. If $F, G$ are $J$-good functors then $F \otimes G$ is $J$-good.

2. If $F$ is a $J$-good functor then $\Delta F$ is $J$-good.

Proof. The first statement follows by tensoring injective resolutions of $F, G$ which are of the form supplied by Theorem 5.0.1. Since $I_{V_{m}} \otimes I_{V_{n}}$ is isomorphic to $I_{V_{m+n}}$, the result then follows by a second application of Theorem 5.0.1.

For the second statement, one applies $\Delta$ to an injective resolution of $F$, as supplied by the theorem. Since $\Delta$ is an exact functor and $\Delta I_{V_{n}}$ is a finite direct sum of $I_{V_{n}}$ 's, the result follows, as before.

Recall that $(G J)_{0} \mathcal{F}$ is taken to denote the Grothendieck group of $J$-good functors. This abelian group has a product which is induced by the tensor product; namely $[F] .[G]=[F \otimes G]$, which gives $(G J)_{0} \mathcal{F}$ a ring structure. The additive identity is [0], whereas the multiplicative identity is $[\mathbb{F}]$.

Remark 5.0.6. The author intends to study the structure of this ring in a future paper; at present this is not determined.

5.1. Example. The example below continues the study of the structure of $I_{V_{3}}$, when $\mathbb{F}=\mathbb{F}_{2}$, started in Section 4 . The reader is referred to this section for the list of simple functors $F_{\lambda}$ with $\lambda_{1} \leq 3$. The above results show that the functor $\bar{D}(2) \otimes \bar{I}$ is $J$-good and it is a sub-functor of $I_{V_{3}}$. It may be studied in the Grothendieck ring $(G J)_{0} \mathcal{F}$; one must find the multiplicity of each co-Weyl object.

Notation 5.1.1. If $F \in \mathcal{F}$, then write $[F] \sim \sum a_{\lambda}\left[F_{\lambda}\right]$, for positive integers $a_{\lambda}$, if there is equality in the Grothendieck ring $G_{0} \mathcal{F}$, modulo factors $F_{\mu}$ with $\mu_{1} \geq 4$.

To perform the calculation it is necessary to calculate (up to the relation $\sim$ ) the functors $F_{\lambda} \otimes F_{\mu}$ for the simple functors appearing above. This may be done, giving the following:

$$
\begin{aligned}
{\left[\Lambda^{1} \otimes \Lambda^{2}\right] } & \sim\left[\Lambda^{3}\right]+\left[F_{(2,1)}\right] \\
{\left[\Lambda^{2} \otimes \Lambda^{2}\right] } & \sim 2\left[F_{(3,1)}\right]+\left[\Lambda^{2}\right] \\
{\left[\Lambda^{3} \otimes \Lambda^{2}\right]=\left[\Lambda^{2} \otimes \Lambda^{3}\right] } & \sim\left[F_{(3,2)}\right] \\
{\left[\Lambda^{1} \otimes \Lambda^{3}\right] } & \sim\left[F_{(3,1)}\right] \\
{\left[\Lambda^{3} \otimes \Lambda^{3}\right] } & \sim\left[\Lambda^{3}\right] \\
{\left[\Lambda^{1} \otimes F_{(3,1)}\right] } & \sim\left[F_{(3,1)}\right]+2\left[F_{(3,2)}\right] \\
{\left[\Lambda^{2} \otimes F_{(3,1)}\right] } & \sim\left[\Lambda^{3}\right]+\left[F_{(3,2,1)}\right] \\
{\left[\Lambda^{3} \otimes F_{(3,1)}\right] } & \sim\left[F_{(3,1)}\right]
\end{aligned}
$$

For this, a number of techniques are used. For the tensor product of two exterior powers, use of the functor $\nabla_{2}$ is invaluable. For the remaining cases, one may study the $G L_{3}$-module obtained by evaluating on $\mathbb{F}^{3}$; the given decompositions then become clear. One concludes, by 'subtracting' the composition factors which occur in $J_{(2)}$ and $J_{(2,1)}$, that:

$$
[\bar{D}(2) \otimes \bar{I}]=\left[J_{(2)}\right]+\left[J_{(2,1)}\right]+2\left[J_{(3)}\right]+3\left[J_{(3,1)}\right]+3\left[J_{(3,2)}\right]+\left[J_{(3,2,1)}\right] .
$$




\section{Proof of The Simplicity THEOREM}

This section is devoted to the proof of the theorem below; throughout the section, $\mathbb{F}$ is taken to be $\mathbb{F}_{2}$, the prime field with two elements. Recall that an analytic functor $F$ is said to be $\tilde{\nabla}_{n}$-nilpotent if there is some $k$ so that $\left(\tilde{\nabla}_{n}\right)^{k} F=0$.

Theorem 6.0.1. Suppose that $\mathbb{F}=\mathbb{F}_{2}$ and that $\lambda$ is a 2-regular partition with $\lambda_{1}=n$. If $F$ is a proper sub-functor of $J_{\lambda}$ then $F$ is $\tilde{\nabla}_{n}$-nilpotent.

The result is established by proving the stronger result:

Proposition 6.0.2. Suppose that $J_{\lambda}$ is as above. If $f: J_{\lambda} \rightarrow G$ is a non-trivial map, then there exists an integer $k$ so that $\left(\tilde{\nabla}_{n}\right)^{k} f$ is injective.

Proof of Theorem 6.0.1 (Assuming Proposition 6.0.2). If $F$ is a proper sub-functor of $J_{\lambda}$ then one may form the quotient map $J_{\lambda} \rightarrow J_{\lambda} / F$, which is non-trivial, by the hypothesis. Thus, Proposition 6.0.2 shows that there exists some $k$ so that $\left(\tilde{\nabla}_{n}\right)^{k} f$ is injective. Consider the sequence of maps:

$$
\left(\tilde{\nabla}_{n}\right)^{k} F \rightarrow\left(\tilde{\nabla}_{n}\right)^{k} J_{\lambda} \rightarrow\left(\tilde{\nabla}_{n}\right)^{k}\left(J_{\lambda} / F\right) .
$$

The first map is injective, since $\tilde{\nabla}_{n}$ preserves injections, and the second map is injective by the choice of $k$. However, the composite is zero, so that it follows that $\left(\tilde{\nabla}_{n}\right)^{k} F=0$, as required.

The proof of Proposition 6.0.2 is facilitated by calculating $\tilde{\nabla}_{n} J_{\lambda}$. This may be done, using the understanding of $J$-good functors which has already been established.

Lemma 6.0.3. If $\lambda$ is a 2-regular partition with $\lambda_{1}=n$ and $\lambda \neq\langle n\rangle$, then $\tilde{\nabla}_{n} J_{\lambda}\left(\mathbb{F}^{n-1}\right)=0$.

Proof. Recall that $J_{\lambda}\left(\mathbb{F}^{n}\right) \cong F_{\lambda}\left(\mathbb{F}^{n}\right)$ and consider the short exact sequence $F_{\lambda} \rightarrow$ $J_{\lambda} \rightarrow J_{\lambda} / F_{\lambda}$. Applying $\tilde{\nabla}_{n}$ yields a short exact sequence

$$
\tilde{\nabla}_{n} F_{\lambda} \rightarrow \tilde{\nabla}_{n} J_{\lambda} \rightarrow C,
$$

in which $C$ is a quotient of $\Delta\left(J_{\lambda} / F_{\lambda}\right)$. Since $\left(J_{\lambda} / F_{\lambda}\right)\left(\mathbb{F}^{n}\right)=0$, it follows that $\Delta\left(J_{\lambda} / F_{\lambda}\right)\left(\mathbb{F}^{n-1}\right)=0$, which gives $C\left(\mathbb{F}^{n-1}\right)=0$. Hence

$$
\tilde{\nabla}_{n} J_{\lambda}\left(\mathbb{F}^{n-1}\right) \cong \tilde{\nabla}_{n} F_{\lambda}\left(\mathbb{F}^{n-1}\right) ;
$$

the latter is zero, by the results of $[\mathrm{P}]$ on the action of $\tilde{\nabla}_{n}$ on simple functors, unless $\lambda=\langle n\rangle$, in which case it is $F_{\bar{\lambda}}\left(\mathbb{F}^{n-1}\right)$.

Proposition 6.0.4. Suppose that $\lambda$ is a 2-regular partition with $\lambda_{1}=n$. If $\lambda=$ $\langle n\rangle$, then $J_{\langle n\rangle} \cong L(n)$ and $\tilde{\nabla}_{n} L(n)=L(n) \oplus L(n-1)$. Otherwise $\tilde{\nabla}_{n} J_{\lambda}=J_{\lambda}$.

The proof uses the following lemma, which follows from Corollary 3.1.3:

Lemma 6.0.5. Suppose that $J_{\lambda}$, for $\lambda_{1}=n$, is a sub-quotient of a sub-functor $F$ of $I_{V_{n}}$; then this sub-quotient $J_{\lambda}$ occurs as a sub-quotient of $k_{n-1} F \subset \widetilde{I_{V_{n}}}$

Proof of Proposition 6.0.4. One starts by establishing that $J_{\lambda}$ is a sub-functor of $\tilde{\nabla}_{n} J_{\lambda}$. Recall that, by construction, $\tilde{\nabla}_{n} J_{\lambda}$ is a quotient of $\Delta J_{\lambda}$, which is $J$-good, by Corollary 5.0.5. Also, $\Delta J_{\lambda}\left(\mathbb{F}^{n-2}\right)=0$, so that $\Delta J_{\lambda}$ only has $J$-factors of the form $J_{\nu}$, with $\nu_{1} \in\{n, n-1\}$. Hence, $\Delta J_{\lambda}$ occurs as an extension

$$
K \rightarrow \Delta J_{\lambda} \rightarrow H,
$$


in which $K$ is $J^{n}$-good and $H$ is $J^{n-1}$-good. Since $\tilde{\nabla}_{n} J_{\lambda}$ is not $\tilde{\nabla}^{n}$-nilpotent, it follows from Corollary 3.1.3 that the map $K \rightarrow \tilde{\nabla}_{n} J_{\lambda}$ is non-trivial, since otherwise $\tilde{\nabla}_{n} J_{\lambda}$ would be the quotient of a $\tilde{\nabla}_{n}$-nilpotent functor. Now $\tilde{\nabla}_{n} J_{\lambda}$ embeds in $\mathcal{J}_{\lambda} \subset I_{\lambda} \oplus I_{\bar{\lambda}}$, so that the basic properties of the co-Weyl objects show that $J_{\lambda}$ is a sub-functor of $\nabla_{n} J_{\lambda}$. It remains to show that the inclusion $J_{\lambda} \hookrightarrow \tilde{\nabla}_{n} J_{\lambda}$ is an isomorphism (in the case $\lambda \neq\langle n\rangle$ ).

Consider the short exact sequence $J_{\lambda} \rightarrow I_{\lambda} \rightarrow Q_{\lambda}$, in which all the functors are $J$-good. Applying $\tilde{\nabla}_{n}$ gives a sequence (not necessarily exact in the middle):

$$
\tilde{\nabla}_{n} J_{\lambda} \hookrightarrow I_{\lambda} \oplus I_{\bar{\lambda}} \longrightarrow \tilde{\nabla}_{n} Q_{\lambda} .
$$

Since $J_{\lambda} \subset \tilde{\nabla}_{n} J_{\lambda}$, the second map factors through $\left(I_{\lambda} / J_{\lambda}\right) \oplus I_{\bar{\lambda}}$.

Now, $Q_{\lambda}$ is a sub-functor of $\bigoplus_{\text {finite }} I_{V_{n-1}}$, so that $\tilde{\nabla}_{n} Q_{\lambda}$ is a sub-functor of $\bigoplus_{\text {finite }} I_{V_{n-1}}$; hence, Lemma 6.0.5 may be applied. If $J_{\mu}$ (for $\mu_{1}=n$ ) is a $J$-factor of $Q_{\lambda}$ with multiplicity $\beta_{\mu}$, then it follows that $J_{\mu}$ occurs as a sub-quotient of $k_{n-1}\left(\tilde{\nabla}_{n} Q_{\lambda}\right)$ with multiplicity $\beta_{\mu}$.

I claim that the induced map $\widetilde{I_{\lambda}} / J_{\lambda} \rightarrow \tilde{\nabla}_{n} Q_{\lambda}$ is injective; write $G$ for the image of this map, which is $J$-good, since the image of any map from a $J^{n}$-good functor to $\bigoplus_{\text {finite }} I_{V_{n}}$ is $J$-good.

To prove injectivity, pass to the quotient map $\left(I_{\lambda} / \widetilde{I}_{\lambda}\right) \rightarrow\left(\tilde{\nabla}_{n} Q_{\lambda}\right) / G$. The domain is a sub-functor of $\bigoplus_{\text {finite }} I_{V_{n-1}}$, hence does not contain a sub-quotient $J_{\mu}$, when $\mu_{1}=n$. Thus, $G$ must contain $J_{\mu}$ with multiplicity $\beta_{\mu}$. Now, by definition, $\beta_{\mu}$ is equal to the multiplicity of $J_{\mu}$ in $\widetilde{I}_{\lambda} / J_{\lambda}$, so this establishes the claim.

Finally, since $\tilde{\nabla}_{n} J_{\lambda}$ embeds in $\widetilde{I}_{\lambda}$, by Lemma 6.0 .3 , and the map $\widetilde{I}_{\lambda} / J_{\lambda} \rightarrow \tilde{\nabla}_{n} Q_{\lambda}$ is injective, it follows that $J_{\lambda}=\tilde{\nabla}_{n} J_{\lambda}$, as required.

Proof of Proposition 6.0.2. The case $\lambda \neq\langle n\rangle$ is treated; the remaining case is obtained by a straightforward adaptation of the argument.

Reduce to considering maps $g: J_{\lambda} \rightarrow I_{W}$ with $\operatorname{dim}(W) \geq n$ as follows; if $f: J_{\lambda} \rightarrow G$ is a non-trivial map then there exists a map $G \rightarrow I_{W}$ so that the composite $J_{\lambda} \rightarrow G \rightarrow I_{W}$ is non-trivial, since the image of $f$ is an analytic functor. Moreover, one can take $\operatorname{dim}(W) \geq n$, by the defining properties of $J_{\lambda}$.

One may fix a standard inclusion $\iota_{\lambda}$ of $J_{\lambda}$ in $I_{V_{n}}$ as follows; namely, take $\iota_{\lambda}$ to be the composite $J_{\lambda} \hookrightarrow \widetilde{I_{V_{n}}} \rightarrow I_{V_{n}}$, where the first map is induced from a surjection $\mathbb{F}\left[G L_{n}\right] \rightarrow S_{\lambda}$ of $G L_{n}$-modules by applying the functor $\operatorname{Hom}_{G L_{n}}\left(-\widetilde{I_{V_{n}}}\right)$. Proposition 6.0.4 shows that $\tilde{\nabla}_{n} J_{\lambda} \cong J_{\lambda}$ (since $\lambda \neq\langle n\rangle$ ); an application of Theorem 2.1.2 shows that the map $\tilde{\nabla}_{n}\left(\iota_{\lambda}\right)$ may be identified with $\iota_{\lambda}$.

Form an extension of $g$ along the map $J_{\lambda} \stackrel{\iota_{\lambda}}{\longrightarrow} I_{V_{n}}$, using the injectivity of $I_{W}$. This gives a commutative diagram:

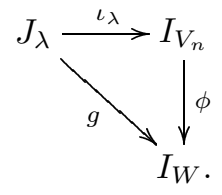

Any map $\phi \in \operatorname{Hom}_{\mathcal{F}}\left(I_{V_{n}}, I_{W}\right)$ may be decomposed uniquely as follows, using the isomorphism $\operatorname{Hom}_{\mathcal{F}}\left(I_{V_{n}}, I_{W}\right) \cong \mathbb{F}\left[\operatorname{hom}\left(V_{n}, W\right)\right]$. Namely, take the decomposition $\operatorname{hom}\left(V_{n}, W\right) \cong \operatorname{hom}_{<n}\left(V_{n}, W\right) \amalg \operatorname{rank}_{n}\left(V_{n}, W\right)$. The set $\operatorname{rank}_{n}\left(V_{n}, W\right)$ decomposes as the union of free right $G L_{n}$-sets, with one orbit for each $n$-plane in $W$; the set of 
$n$-planes in $W$ is written as $\operatorname{Gr}_{n}(W)$, corresponding to the Grassmannian. Then, if $\phi \in \operatorname{Hom}_{\mathcal{F}}\left(I_{V_{n}}, I_{W}\right)$, one may write

$$
\phi=\phi_{0}+\sum_{\pi \in \operatorname{Gr}_{n}(W)} \phi_{\pi},
$$

where $\phi_{0} \in \mathbb{F}\left[\operatorname{hom}_{<n}\left(V_{n}, W\right)\right]$ and each $\phi_{\pi}$ is the sum of rank $n$ maps which are in the conjugacy class of $\pi$. The importance of this approach is shown by the following facts, which follow from the defining properties of $J_{\lambda}$ :

1. $\phi_{0}$ is zero when restricted to $J_{\lambda}$.

2. A map $\phi_{\pi}: I_{V_{n}} \rightarrow I_{W}$ is either injective or zero when restricted to $J_{\lambda}$.

The second point follows by observing that a map $\phi_{\pi}$ may be factored as $I_{V_{n}} \rightarrow$ $I_{V_{n}} \rightarrow I_{W}$, in which the second map is an injection.

Define the $\lambda$-length of a map $\phi: I_{V_{n}} \rightarrow I_{W}$ (with respect to $\iota_{\lambda}$ ) to be the number of $\pi \in \mathrm{Gr}_{\mathrm{n}}(W)$ such that $J_{\lambda} \stackrel{\iota_{\lambda}}{\hookrightarrow} I_{V_{n}} \stackrel{\phi_{\pi}}{\longrightarrow} I_{W}$ is non-zero (hence injective).

The proof of the proposition is by induction on the $\lambda$-length of $\phi$. If $\phi$ has $\lambda$ length 1 , where $g=\left.\phi\right|_{J_{\lambda}}$, then $g$ is necessarily injective, so that it suffices to take $k=0$.

For the inductive step, suppose that the $\lambda$-length of $\phi$ is greater than one and consider the map $\nabla_{n} \phi: I_{V_{n}} \cong \nabla_{n} I_{V_{n}} \rightarrow \Lambda^{n}(W) \otimes I_{W} \cong \nabla_{n}\left(I_{W}\right)$. This map identifies with

$$
\sum_{\pi \in \operatorname{Gr}_{n}(W)} \mathbf{v}_{\pi} \otimes \phi_{\pi},
$$

where $\mathbf{v}_{\pi} \in \Lambda^{n}(W)$ is the image under $\Lambda^{n}(\pi)$ of the non-zero vector in $\Lambda^{n}\left(\mathbb{F}^{n}\right)$.

Since the $\lambda$-length of $\phi$ is greater than one, there are $\sigma, \tau \in \operatorname{Gr}_{n}(W)$ so that $\phi_{\sigma} \iota_{\lambda}$ and $\phi_{\tau} \iota_{\lambda}$ are both injective. Since $\sigma, \tau$ are distinct planes in $W$, the vectors $\mathbf{v}_{\sigma}$ and $\mathbf{v}_{\tau}$ are distinct; more particularly, there is a surjection $W \rightarrow \mathbb{F}^{n}$ so that $\Lambda^{n}(\beta) \mathbf{v}_{\sigma} \neq 0$ and $\Lambda^{n}(\beta) \mathbf{v}_{\tau}=0$.

Now, consider the map $\Lambda^{n}(\beta) \otimes I_{W}: \Lambda^{n}(W) \otimes I_{W} \rightarrow I_{W}$, which fits into the commutative diagram:

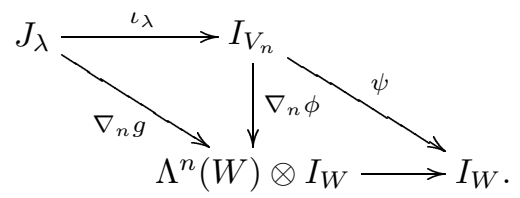

By construction, $\psi=\sum_{\pi \in \operatorname{Gr}_{n}(W) \mid \Lambda^{n}(\beta) \mathbf{v}_{\pi}=1} \phi_{\pi}$; by the choice of $\beta$ it follows that the $\lambda$-length of $\psi$ satisfies $1 \leq \lambda$ - length $\psi<\lambda$ - length $\phi$. Hence, by induction, there is a $k$ so that $\left(\nabla_{n}\right)^{k} \psi$ is injective. Applying $\left(\nabla_{n}\right)^{k}$ to the above diagram shows that $\left(\nabla_{n}\right)^{k+1} g$ is injective, which is the required result.

Recall that there is a unique non-trivial map $J_{\lambda} \hookrightarrow I_{\lambda}$.

Corollary 6.0.6. Suppose that $I_{\lambda} \stackrel{f}{\rightarrow} G$ is a map so that the composite $g: J_{\lambda} \hookrightarrow$ $I_{\lambda} \rightarrow G$ is non-trivial. There exists a $k$ so that $\left(\nabla_{n}\right)^{k} f$ is injective.

Proof. We may assume that $\lambda \neq\langle n\rangle$, since $I_{\lambda}=L(n)=J_{\langle n\rangle}$ in this case. Proposition 6.0.2 implies that there is a $k$ so that $\left(\nabla_{n}\right)^{k-1} g$ is injective; now $\left(\nabla_{n}\right)^{k-1} g$ factors through $\left(\nabla_{n}\right)^{k-1} I_{\lambda} \cong I_{\lambda} \oplus I_{\bar{\lambda}}$ and $\left(\nabla_{n}\right)^{k-1}$ of the inclusion $J_{\lambda} \rightarrow I_{\lambda}$ must identify with the unique inclusion $J_{\lambda} \hookrightarrow I_{\lambda} \oplus I_{\bar{\lambda}}$. It follows that $\left(\nabla_{n}\right)^{k-1} f$ is injective when restricted to $I_{\lambda}$. 
To complete the proof, observe that $\nabla_{n}$ of the inclusion $I_{\lambda} \hookrightarrow I_{\lambda} \oplus I_{\bar{\lambda}}$ is the identity on $I_{\lambda} \oplus I_{\bar{\lambda}}$. It follows immediately that $\left(\nabla_{n}\right)^{k} f$ is injective.

6.1. Consequences of the simplicity theorem. The full sub-category $\tilde{\nabla}_{n}-\mathcal{N} i l$ $\subset \mathcal{F}_{\omega}$ of functors which are nilpotent under $\tilde{\nabla}_{n}$ is shown to be thick in $[\mathrm{P}]$; thus, one may study the category localized away from the $\tilde{\nabla}_{n}$-nilpotent functors. For the purposes of this paper, write $\mathcal{C}_{n}$ for the category $\tilde{\nabla}_{n}-\mathcal{N} i l$, regarded as a full sub-category of $\mathcal{F}_{\omega}$.

Recall the construction of the quotient category $\mathcal{F}_{\omega} / \mathcal{C}_{n}$, as described in $[\mathrm{G}]$. The objects in the category are the same as those of $\mathcal{F}_{\omega}$ : namely the analytic functors, whereas, for $X, Y \in \mathrm{Ob} \mathcal{F}_{\omega}$ :

$$
\operatorname{Hom}_{\mathcal{F}_{\omega} / \mathcal{C}_{n}}(X, Y) \cong \lim _{X^{\prime}, Y^{\prime} \rightarrow} \operatorname{Hom}_{\mathcal{F}}\left(X^{\prime}, Y / Y^{\prime}\right)
$$

where the limit is taken over those $X^{\prime} \subset X$ and $Y^{\prime} \subset Y$ such that $X / X^{\prime}$ and $Y^{\prime}$ are objects in $\mathcal{C}_{n}$ (ie they are $\tilde{\nabla}_{n}$-nilpotent). The work of [G, Chapter 3] shows that $\mathcal{F}_{\omega} / \mathcal{C}_{n}$ is an abelian category; moreover, an object $S$ in the quotient category is simple if and only if whenever $A \hookrightarrow S$ is an inclusion in $\mathcal{F}_{\omega}$, either $A$ is in $\mathcal{C}_{n}$ or the cokernel of $A \hookrightarrow S$ is in $\mathcal{C}_{n}$.

\section{Proposition 6.1.1.}

1. The functors $J_{\lambda}$, where $\lambda_{1}=n$, are simple objects in the quotient category $\mathcal{F}_{\omega} / \mathcal{C}_{n}$.

2. If $\lambda_{1}=\mu_{1}=n$, then $J_{\lambda}$ and $J_{\mu}$ are isomorphic in $\mathcal{F}_{\omega} / \mathcal{C}_{n}$ if and only if $\lambda=\mu$.

3. The endomorphism ring of $J_{\lambda}, \operatorname{End}_{\mathcal{F}_{\omega} / \mathcal{C}_{n}}\left(J_{\lambda}\right)$ is $\mathbb{F}$.

Proof. The first statement is a straightforward corollary to Theorem 6.0.1. To prove the second, consider a non-zero map in $\mathcal{F}_{\omega} / \mathcal{C}_{n}$ between $J_{\lambda}$ and $J_{\mu}$. This is represented by a map $F \stackrel{f}{\rightarrow} J_{\mu} / G$, where $F \subset J_{\lambda}, G \subset J_{\mu}$ and where both $J_{\lambda} / F$ and $G$ are $\tilde{\nabla}_{n}$-nilpotent.

Theorem 6.0.1 implies immediately that $F=J_{\lambda}$, using the fact that $J_{\lambda}$ is not $\tilde{\nabla}$-nilpotent and the category $\mathcal{C}_{n}$ is thick. Now apply Proposition 6.0.2 to consider the maps $f: J_{\lambda} \rightarrow J_{\mu} / G$ and the natural surjection $J_{\mu} \rightarrow J_{\mu} / G$; conclude that there exists $k \geq 0$ so that both $\left(\tilde{\nabla}_{n}\right)^{k} f:\left(\tilde{\nabla}_{n}\right)^{k} J_{\lambda} \rightarrow\left(\tilde{\nabla}_{n}\right)^{k}\left(J_{\mu} / G\right)$ is injective and $\left(\tilde{\nabla}_{n}\right)^{k}\left(J_{\mu} / G\right)$ is isomorphic to $\left(\tilde{\nabla}_{n}\right)^{k} J_{\mu}$. Since $\left(\tilde{\nabla}_{n}\right)^{k} J_{\mu}$ always embeds in $I_{\mu} \oplus I_{\bar{\mu}}$, it follows that $\left(\tilde{\nabla}_{n}\right)^{k} f$ induces an injection $\left(\tilde{\nabla}_{n}\right)^{k} J_{\lambda} \hookrightarrow I_{\mu} \oplus I_{\bar{\mu}} ; J_{\lambda}$ is a sub-functor of $\left(\tilde{\nabla}_{n}\right)^{k} J_{\lambda}$, so that it follows that $\lambda=\mu$.

Finally, in the case that $\lambda=\mu$, if $p: J_{\lambda} \rightarrow J_{\lambda} / G$ denotes the natural surjection, then an adaptation of the above argument shows that $\left(\tilde{\nabla}_{n}\right)^{k} f$ identifies with $\left(\tilde{\nabla}_{n}\right)^{k} p$ for $k$ sufficiently large. Thus, $\left(\tilde{\nabla}_{n}\right)^{k}(f-p)$ is zero, which implies that $f=p$, by Proposition 6.0.2. The natural surjections $J_{\lambda} \rightarrow J_{\lambda} / G$, as $G$ varies amongst proper sub-functors of $J_{\lambda}$ all represent the same homomorphism in the localized category. This completes the proof of the result.

Remark 6.1.2. These simple objects in $\mathcal{F}_{\omega} / \mathcal{C}_{n}$ are far from being the only simple objects in the quotient category. For example, the results of [P1] construct a nonfinite family of simple objects in the category $\mathcal{F}_{\omega} / \mathcal{C}_{1}$, which occur as sub-functors of $\bar{I} \otimes \Lambda^{t}$ as $t$ varies. 


\section{Boolean ALGeBras AND MOdUle STRUCTURES}

The purpose of this section is to show that many $J$-good functors arise naturally; let $\mathcal{B}$ denote the category of $\mathbb{F}$-Boolean algebras. $\mathcal{B}$ is equivalent to the opposite of the category $\mathcal{P S}$ of profinite sets: if $B$ is an $\mathbb{F}$-Boolean algebra, then write spec $B$ for the set of algebra maps $\operatorname{Alg}(B, \mathbb{F})$. This is a profinite set, since $B$ is the colimit of its finitely-generated sub-algebras. There is a natural isomorphism $\mathbb{F}^{\text {Spec } B}$, where this is the set of continuous set maps from $\operatorname{Spec} B$ to $\mathbb{F}$.

Write $\mathcal{B}^{\mathcal{E}_{f}}$ for the category of functors $\mathcal{E}_{f} \rightarrow \mathcal{B}$; this is regarded as a sub-category of $\mathcal{F}$, namely the category of Boolean algebra valued functors. This category is of fundamental interest due to the connection with the study of unstable algebras over the Steenrod algebra, as established by [HLS, Part II].

There is an equivalence of categories between $\mathcal{B}^{\mathcal{E}_{f}}$ and $\operatorname{Func}\left\{\mathcal{E}_{f}^{o p} \rightarrow \mathcal{P S}\right\}$. Suppose that $\gamma: \mathcal{E}_{f}^{o p} \rightarrow \mathcal{P S}$ is such a functor; the associated functor in $\mathcal{F}$ is given by $V \mapsto \mathbb{F}^{\gamma(V)}$.

There is an elegant translation of the notion of transcendence degree for an algebra to the category $\mathcal{B}^{\mathcal{E}_{f}}$, which is established in [HLS]. For the purposes of this paper, the following restricted notion is sufficient.

Definition 7.0.1. A functor $K \in \mathcal{B}^{\mathcal{E}_{f}}$ which takes finite-dimensional values has transcendence degree $\leq d$ if $K \cong R_{d}\left(\mathbb{F}^{S}\right)$, for some finite right $\operatorname{End}\left(V_{d}\right)$-set $S$. Equivalently, $K$ is represented by the functor $\mathcal{E}_{f}^{o p} \rightarrow \mathcal{P} \mathcal{S}: V \mapsto S \times_{\operatorname{End}\left(V_{d}\right)} \operatorname{hom}\left(V, V_{d}\right)$.

Example 7.0.2. Examples of Boolean algebra functors of transcendence degree $\leq d$ have already appeared in this paper; the functors $I_{\mathbb{F}^{d}}, \widetilde{I_{\mathbb{F}^{d}}} \oplus \mathbb{F}$ and $\bar{D}(d) \oplus \mathbb{F}$ may all be regarded as such functors.

$I_{\mathbb{F}^{d}}$ is represented by the right $\operatorname{End}\left(V_{d}\right)$-set $\operatorname{hom}\left(\mathbb{F}^{d}, V_{d}^{*}\right)$, where the $\operatorname{End}\left(V_{d}\right)$ action is the transpose of the usual action on hom $\left(\mathbb{F}^{d}, V_{d}\right) . \mathbb{F} \oplus \widetilde{I_{\mathbb{F}^{d}}}$ is represented by the $\operatorname{End}\left(V_{d}\right)$-set which is obtained by collapsing all the singular endomorphisms to a point in the above set. Hence, this identifies with $* \amalg G L_{d}$, with an $\operatorname{End}\left(V_{d}\right)$ action which is induced by the above quotient. Finally, $\mathbb{F} \oplus \bar{D}(d)$ is represented by the smallest possible non-trivial $\operatorname{End}\left(V_{d}\right)$-set, namely $X_{d}=*_{0} \amalg *_{1}$, where $*_{0}$ is a sub $\operatorname{End}\left(V_{d}\right)$-set.

The functor $I_{\mathbb{F}^{d}}$ is injective in the category $\mathcal{B}^{\mathcal{E}_{f}}$ and is determined by the representing property that follows: if $\gamma$ is a functor $\mathcal{E}_{f}^{o p}$ which represents $K \in \mathcal{B}^{\mathcal{E}_{f}}$, then $\operatorname{Hom}_{\mathcal{B}^{\mathcal{E}_{f}}}\left(K, I_{\mathbb{F}^{d}}\right) \cong \gamma\left(\mathbb{F}^{d *}\right)$.

This allows the statement of the main result of this section:

Theorem 7.0.3. Suppose that $K \in \mathcal{B}^{\mathcal{E}_{f}}$ takes finite-dimensional values and has finite transcendence degree, then $K$ is $J$-good as a functor in $\mathcal{F}$.

Proof. The proof is by induction upon $d$, the transcendence degree of $K$. The case $d=0$ is easy, since the functor is then a constant functor (with Boolean algebra structure) and is therefore $J$-good. The first step in the proof is to observe that we may reduce to the case $K(0)=\mathbb{F}$ and perform the induction on such functors.

Suppose that the result holds for $L \in \mathcal{B}^{\mathcal{E}_{f}}$, with $L(0)=\mathbb{F}$ and the transcendence degree of $L$ is less than $d$ and consider $K$ with transcendence degree exactly $d$. Hence, there is some minimal $k \leq d$ so that $K\left(\mathbb{F}^{k}\right) \neq K(0)$. 
In the case that $k=d$ and the $\operatorname{End}\left(V_{d}\right)$-set representing $K$ is $S$, then $\mathbb{F}^{S} \cong \mathbb{F} \oplus \overline{\mathbb{F}^{S}}$ as an $\operatorname{End}\left(V_{d}\right)$-module, where every singular endomorphism acts trivially on $\overline{\mathbb{F}^{S}}$. Hence $R_{d}\left(\mathbb{F}^{S}\right) \cong \mathbb{F} \oplus R_{d}\left(\overline{\mathbb{F}^{S}}\right)$ is $J$-good, by Proposition 2.2.1.

This allows an induction on the number of elements in $S$ : as hypothesis, suppose that the theorem is proved for all $K$ induced from $\operatorname{End}\left(V_{d}\right)$-sets with strictly fewer than $N$ elements. The initial stage of the induction is the trivial case when $N=1$.

Now suppose that $|S|=N$ and consider the number $k$ defined above; the case $k=d$ is covered by the previous discussion. If $k<d$, then there is a non-trivial map in $\mathcal{B}^{\mathcal{E}_{f}}, \alpha: K \rightarrow I_{\mathbb{F}^{k}}$, which allows one to perform the inductive step on $N$.

The kernel $L$ of $\alpha$ is a functorial Boolean algebra ideal, so that $\mathbb{F} \oplus L$ is naturally an object in $\mathcal{B}_{f}^{\mathcal{E}}$. Since the map $\alpha$ is of the form $R_{d}\left(\mathbb{F}^{S}\right) \rightarrow R_{d}\left(\operatorname{hom}\left(\mathbb{F}^{k}, V_{d}^{*}\right)\right)$, it follows that $\mathbb{F} \oplus L \cong R_{d}\left(\mathbb{F}^{T}\right)$, for some $\operatorname{End}\left(V_{d}\right)$-set $T$ with $|T|<|S|$, using the left exactness of $R_{d}$. Hence, by the inductive hypothesis, $\mathbb{F} \oplus L$ (and hence $L$ ) is $J$-good.

The image $A$ of the map $\alpha: K \rightarrow I_{\mathbb{F}^{k}}$ is a Boolean algebra valued functor; it is a standard argument (essentially by Yoneda's lemma) to show that $A \subset I_{\mathbb{F}^{k}}$ has transcendence degree $\leq k$, Thus, by induction, $A$ is $J$-good, using the fact that $k<d$. It follows that $K$ is $J$-good, completing the induction upon $N$ and thus the induction upon $d$.

This theorem provides us with evidence that $J$-good functors occur naturally in the study of the category $\mathcal{F}$, since the Boolean algebra valued functors have an importance stressed by [HLS].

The following result may be compared with the results of Henn, $[\mathrm{H}]$, who works entirely within the category of unstable modules over the Steenrod algebra, in a more general situation.

Corollary 7.0.4. Every object $K \in \mathcal{B}^{\mathcal{E}_{f}}$ which takes finite-dimensional values and which has finite transcendence degree $\leq d$ has an injective resolution $0 \rightarrow K \rightarrow I^{\bullet}$, in which each $I^{\bullet}$ is a direct summand of a finite direct sum $\bigoplus I_{V_{d}}$.

Proof. This follows directly by combining Theorem 7.0.3 with the easier implication of Theorem 5.0.1.

Example 7.0.5. Consider the Dickson algebra functor $D(n):=\left(I_{\mathbb{F}^{n}}\right)^{G L_{n}}$. This is a Boolean algebra valued functor which may be seen to be represented by the $\operatorname{End}\left(V_{n}\right)$-set hom $\left(\mathbb{F}^{n}, V_{n}^{*}\right) / G L_{n}$. This set is filtered by the rank of the maps (as an $\operatorname{End}\left(V_{n}\right)$-set). The set of points represented by a map of rank $k$ is

$$
\operatorname{rank}_{k}\left(\mathbb{F}^{n}, V_{n}^{*}\right) / G L_{n}
$$

which identifies with $\operatorname{Gr}_{k}\left(V_{n}^{*}\right)$, the set of $k$-planes in $V_{n}^{*}$. This corresponds to the fact that the functor $D(n) / \mathbb{F}$ admits a unique $J$-good filtration, in which $\bar{D}(k)$ occurs once, for $1 \leq k \leq n$. The factor $\mathbb{F} \cong \bar{D}(0)$ occurs as a direct summand of the functor $D(n)$.

7.1. Module structures for the $J_{\lambda}$. Throughout this section, it is supposed that $\mathbb{F}=\mathbb{F}_{2}$, the prime field with two elements. In this case, $\Lambda^{n}\left(\mathbb{F}^{n}\right)$ is isomorphic to the trivial $G L_{n}$-module $\mathbb{F}$; thus, for any simple $G L_{n}$-module $S_{\lambda}$, there is an isomorphism in $\mathcal{G} \mathcal{L}_{n}, \Lambda^{n}\left(\mathbb{F}^{n}\right) \otimes S_{\lambda} \cong S_{\lambda}$. Recall that the co-Weyl object $J_{\lambda}$ is defined as $R_{n}\left(J_{\lambda}\right)$; it follows that there is a unique non-trivial map $R_{n}\left(\Lambda^{n}\left(\mathbb{F}^{n}\right)\right) \otimes R_{n}\left(S_{\lambda}\right) \rightarrow R_{n}\left(S_{\lambda}\right)$, by using the $\left(E_{n}, R_{n}\right)$-adjunction and the fact that $E_{n}$ commutes with the tensor 
product. This argument extends to show that this unique non-trivial map, which identifies as a map $\mu_{\lambda}: \bar{D}(n) \otimes J_{\lambda} \rightarrow J_{\lambda}$ fits into a commutative diagram:

$$
\begin{array}{ccc}
\bar{D}(n) \otimes \bar{D}(n) \otimes J_{\lambda} & \stackrel{\bar{D}(n) \otimes \mu_{\lambda}}{\longrightarrow} & \bar{D}(n) \otimes J_{\lambda} \\
\mu \otimes J_{\lambda} \downarrow & & \downarrow \mu_{\lambda} \\
\bar{D}(n) \otimes J_{\lambda} & \stackrel{\mu_{\lambda}}{\longrightarrow} & J_{\lambda} ;
\end{array}
$$

this shows that $J_{\lambda}$ is a $\bar{D}(n)$-module, in an obvious sense, since the product $\bar{D}(n) \otimes$ $\bar{D}(n) \rightarrow \bar{D}(n)$ is the restriction of the Boolean algebra structure on $\mathbb{F} \oplus \bar{D}(n)$. This product may also be described as the restriction of the product on $I_{V_{n}}$.

One may consider the composite $\bar{D}(n) \otimes J_{\lambda} \hookrightarrow I_{V_{n}} \otimes I_{V_{n}} \rightarrow I_{V_{n}}$, induced by inclusions of $\bar{D}(n)$ and $J_{\lambda}$ into $I_{V_{n}}$ and where $I_{V_{n}} \otimes I_{V_{n}} \rightarrow I_{V_{n}}$ is the product on $I_{V_{n}}$. It is clear that $\left\{\bar{D}(n) \otimes J_{\lambda}\right\}\left(\mathbb{F}^{n}\right) \cong S_{\lambda}$; this implies that the above map factors through some map $J_{\lambda} \hookrightarrow I_{V_{n}}$. Hence, the above discussion has proved:

Proposition 7.1.1. Suppose that $S_{\lambda}$ is a simple $G L_{n}$-module. The multiplication on $I_{V_{n}}$ restricts to a map $\bar{D}(n) \otimes J_{\lambda} \rightarrow J_{\lambda}$, which is a map of $\bar{D}(n)$-modules.

Corollary 7.1.2. Suppose that $S_{\lambda}$ is a simple $G L_{n}$-module. There is a canonical surjection $\bar{D}(n) \otimes F_{\lambda} \rightarrow J_{\lambda}$.

Proof. There is certainly a unique non-trivial map given by the above constructions $\bar{D}(n) \otimes F_{\lambda} \rightarrow J_{\lambda}$, so that it is sufficient here to show that this map is surjective. Since the image is a sub-functor of $J_{\lambda}$, Theorem 6.0 .1 shows that it is sufficient to show that the image is not nilpotent under the action of $\tilde{\nabla}_{n}$. This is shown by using the description of this map given above and the fact that the product map induces an injection $I_{V_{n}}(V) \otimes I_{V_{n}}(W) \hookrightarrow I_{V_{n}}(V \oplus W)$. Hence, the following composite is injective:

$$
\bar{D}(n)(V) \otimes F_{\lambda}(W) \rightarrow\left(\bar{D}(n) \otimes F_{\lambda}\right)(V \oplus W) \rightarrow I_{V_{n}}(V \oplus W) .
$$

This allows one to deduce that the image is not $\tilde{\nabla}_{n}$-nilpotent, since $\bar{D}(n)$ is not nilpotent. Essentially, one applies $\left(\tilde{\nabla}_{n}\right)^{t}$ with respect to a splitting of $V$ as $V^{\prime} \oplus$ $\mathbb{F}^{t}$.

Remark 7.1.3. The above results show that the structure of the co-Weyl functor $J_{\lambda}$ induced from $S_{\lambda}$ is intimately related to the structure of $\bar{D}(n)$. This is further evidence that the functor $\bar{D}(n)$ plays a fundamental rôle in the category $\mathcal{F}$.

\section{ACKNOWLEDGEMENT}

The author would like to thank Lionel Schwartz and Nick Kuhn for initiating him to the category $\mathcal{F}$, for many conversations related to this subject area and also for their comments on a preliminary version of this paper ${ }^{1}$. Finally, the author is grateful for the comments made by the referee and their careful reading of the text.

\section{REFERENCES}

[CPS] E. Cline, B. Parshall and L. Scott, Finite dimensional algebras and highest weight categories, J. Reine angew. 391 (1988), 85-99. MR 90d:18005

[CR] C.W. Curtis and I. Reiner, Methods of Representation Theory, Volume I, (1981), John Wiley and Sons. MR 90k:20001

\footnotetext{
${ }^{1}$ This paper is a substantially reorganized version of the preprint which appeared as a Prépublication en Mathématiques de l'Univeristé de Paris-Nord, in 1996.
} 
[EM] S. Eilenberg and S. Mac Lane, On the groups $H(\Pi, n)$, II, Ann. Math. 60, (1954), 49-139. MR 16:392

[FLS] V. Franjou, J. Lannes and L. Schwartz, Autour de la cohomologie de Mac Lane des corps finis, Invent. Math. 115 (1994), 513-538. MR 95d:19002

[FP] E.M. Friedlander and B. Parshall, Cohomology of Lie algebras and algebraic groups, Amer. J. Math. 108 (1986), 235-253. MR 87c:20080

[G] P. Gabriel, Des catégories abéliennes, Bull. Soc. Math. France 90 (1962), 323-448. MR 38:1144

[HHS] J.C. Harris, T. Hunter and R.J. Shank, Steenrod algebra module maps from $H^{*}\left(B(Z / p)^{n}\right)$ to $H^{*}\left(B(Z / p)^{s}\right)$, Proc. Amer. Math. Soc. 112 (1991), 245-257. MR 91i:55023

[HK] J.C. Harris and N.J. Kuhn, Stable decompositions of classifying spaces of finite abelian p-groups, Math. Proc. Camb. Phil. Soc. 103 (1988), 427-449. MR 89d:55021

[HS] J.C. Harris and R.J. Shank Lannes, T-functor on summands of $H^{*}\left(B(\mathbf{Z} / p)^{s}\right)$, Trans. Amer. Math. Soc. 333 (1992), 579-606. MR 92m:55024

[H] H.-W. Henn, Finiteness properties of injective resolutions of certain unstable modules over the Steenrod algebra and applications, Math. Ann. 291 (1991), 191-203. MR 92i:55016

[HLS] H.-W. Henn, J. Lannes and L. Schwartz, The categories of unstable modules and unstable algebras over the Steenrod algebra modulo nilpotent objects, Amer. J. Math. 115 (1993), 1053-1106. MR 94i:55024

[J] J.C. Jantzen, Representations of Algebraic Groups, Volume 131, Pure. Appl. Math., Academic Press, (1987). MR 89c:20001

[KK] P. Krason and N.J. Kuhn, On embedding polynomial functors in symmetric powers, $J$. Algebra 163, (1994), 281-294. MR 95e:20058

[K1] N.J. Kuhn, Generic representations of the finite general linear groups and the Steenrod algebra: I, Amer. J. Math. 116 (1993), 327-360. MR 95c:55022

[K2] N.J. Kuhn, Generic representations of the finite general linear groups and the Steenrod algebra: II, K-Theory 8 (1994), 395-426. MR 95k:55038

[K3] N.J. Kuhn, Generic representations of the finite general linear groups and the Steenrod algebra: III, K-Theory 9 (1995), 273-303. MR 97c:55026

[K4] N.J. Kuhn, On the Morava K-theories of some classifying spaces, Trans. Amer. Math. Soc. 304 (1987), 285-298. MR 89d:55013

[L] J. Lannes, Sur les espaces fonctionnels dont la source est le classifiant d'un $p$-groupe abélien élémentaire, Publ. I.H.E.S. 75 (1992), 135-244. MR 93j:55019

[M] S. Martin, Schur Algebras and Representation Theory, Cambridge Tracts in Mathematics 112 (1993), Cambridge University Press. MR 95f:20071

[MP] S. Mitchell and S. Priddy, Stable splittings derived from the Steinberg module, Topology 22 (1983), 285-298. MR 85f:55005

[Pi] L. Piriou, Thèse de doctorat, Université de Paris VII, (1995).

[PS] G.M.L. Powell with an Appendix by L. Schwartz, The Artinian conjecture for $I \otimes I$, To appear, J. Pure Appl. Alg.

[P] G.M.L. Powell, Polynomial filtrations and Lannes' T-functor, K-Theory 13 (1998), 279304. CMP 98:10

[P1] G.M.L. Powell, The structure of $\bar{I} \otimes \Lambda^{n}$ in generic representation theory, J. Alg. 194 (1997), 455-465. CMP 97:17

[S] L. Schwartz, Unstable Modules over the Steenrod Algebra and Sullivan's Fixed Point Set Conjecture, Chicago Lecture Notes in Mathematics, Univ. Chicago Press, Chicago and London (1994). MR 95d:55017

LAGA, Institut Galilée, Université Paris 13, 93430 Villetaneuse, France

E-mail address: powell@math.univ-paris13.fr 